\title{
GEORGE LORENTZ AND INEQUALITIES IN APPROXIMATION
}

\author{
T. ERDÉLYI
}

\begin{abstract}
George Lorentz influenced the author's research on inequalities in approximation in many ways. This is the connecting thread of this survey paper. The themes of the survey are listed at the very beginning of the paper.
\end{abstract}

\section{CONTENTS}

$\S 1 . \quad$ Bernstein-type inequalities for exponential sums 365

§2. Remez-type inequalities for exponential sums $\quad 369$

$\S 3 . \quad$ Lorentz degree of polynomials 372

\begin{tabular}{l|l|} 
§4. Markov- and Bernstein-type inequalities & 372 \\
for polynomials with constraints & 376
\end{tabular}

$\S 5$. Müntz-type theorems $\quad 376$

§6. Remez-type inequalities and Newman's product problem 378

§7. Multivariate approximation $\quad 379$

§8. Newman's inequality 379

§9. Littlewood polynomials 382

§10. Inequalities for generalized polynomials $\quad 384$

§11. Markov- and Bernstein-type inequalities for rational functions 387

§12. Nikolskil-type inequalities for shift-invariant function spaces $\quad 391$

$\S 13 . \quad$ Inverse Markov- and Bernstein-type inequalities $\quad 391$

§14. Ultraflat sequences of unimodular polynomials $\quad 392$

$\S 15$. Zeros of polynomials with restricted coefficients $\quad 396$

References

\section{§1. BERNSTEIN-TYPE INEQUALITIES FOR EXPONENTIAL SUMS}

The results in this section were, in large measure, motivated by the letter of Lorentz below.

"Dear Tamás:

Feb. 27, 1988

I know you are interested in Bernstein-type inequalities and I am also. In some nonlinear cases one has

$$
\left\|P^{\prime}\right\|_{X} \leq \Phi(n)\|P\|_{Y},
$$

where $n$ is the dimension of the set of the $P$ 's, and the norms are taken in different Banach spaces $X$ and $Y$. For instance, inequalities of Dolzhenko and Pekarskii for rational

2000 Mathematics Subject Classification. Primary 41-02.

Key words and phrases. Bernstein-type inequality, Remez-type inequality, Littlewood polynomials, generalized polynomials, multivariate approximation.

(C)2010 American Mathematical Society 
functions are of this type. I have proved an inequality of this type for exponential functions $\sum_{1}^{n} a_{j} e^{\lambda_{j} x}$ or the extended exponential sums

$$
\sum_{j=1}^{l} P_{k_{j}}(x) e^{\lambda_{j} x}, \quad \sum_{1}^{l}\left(k_{j}+1\right)=n .
$$

I enclose my arguments. Would you like to help me? Are my inequalities sharp in some sense? What are other arguments that can lead to such inequalities? Hope to see you again in April, when I will be again in Columbia.

Sincerely, George Lorentz"

In his book [41] Braess writes: "The rational functions and exponential sums belong to those concrete families of functions which are the most frequently used in nonlinear approximation theory. The starting point of consideration of exponential sums is an approximation problem often encountered for the analysis of decay processes in natural sciences. A given empirical function on a real interval is to be approximated by sums of the form

$$
\sum_{j=1}^{n} a_{j} e^{\lambda_{j} t}
$$

where the parameters $a_{j}$ and $\lambda_{j}$ are to be determined, while $n$ is fixed."

In 23] we proved the right Bernstein-type inequality for exponential sums.

Let

$$
E_{n}:=\left\{f: f(t)=a_{0}+\sum_{j=1}^{n} a_{j} e^{\lambda_{j} t}, a_{j}, \lambda_{j} \in \mathbb{R}\right\} .
$$

So $E_{n}$ is the collection of all $(n+1)$-term exponential sums with constant first term. Schmidt [128] proved that there is a constant $c(n)$ depending only on $n$ so that

$$
\left\|f^{\prime}\right\|_{[a+\delta, b-\delta]} \leq c(n) \delta^{-1}\|f\|_{[a, b]}
$$

for every $f \in E_{n}$ and $\delta \in\left(0, \frac{1}{2}(b-a)\right)$. Here, and in what follows, $\|\cdot\|_{[a, b]}$ denotes the uniform norm on $[a, b]$. Lorentz [103] improved Schmidt's result by showing that, for every $\alpha>\frac{1}{2}$, there is a constant $c(\alpha)$ depending only on $\alpha$ so that $c(n)$ in the above inequality can be replaced by $c(\alpha) n^{\alpha \log n}$ (Xu improved this to allow $\alpha=\frac{1}{2}$ ), and he speculated that there may be an absolute constant $c$ so that Schmidt's inequality holds with $c(n)$ replaced by $c n$. The main result of [23, Theorem 3.2, shows that Schmidt's inequality is true with $c(n)=2 n-1$. That is,

$$
\sup _{0 \neq f \in E_{n}} \frac{\left|f^{\prime}(y)\right|}{\|f\|_{[a, b]}} \leq \frac{2 n-1}{\min \{y-a, b-y\}}, \quad y \in(a, b) .
$$

In this Bernstein-type inequality even the pointwise factor is sharp up to a multiplicative absolute constant; the inequality

$$
\frac{1}{e-1} \frac{n-1}{\min \{y-a, b-y\}} \leq \sup _{0 \neq f \in E_{n}} \frac{\left|f^{\prime}(y)\right|}{\|f\|_{[a, b]}}, \quad y \in(a, b),
$$

was established by Theorem 3.3 in 23 . Inequality (1.1) improves an earlier result obtained in [16, where we have only $8 n^{2}$ in place of $2 n-1$. Lorentz presented our simple elegant proof of our weaker result in [16] on pages 378 and 379 of his book [105] and remarked that the exact inequality (1.1) would appear in [17. I do not know if Lorentz ever read my paper [23, but it looks to me that he accepted it as it was presented in my book [17] with P. Borwein.

Bernstein-type inequalities play a central role in approximation theory via a machinery developed by Bernstein, which turns Bernstein-type inequalities into inverse theorems of 
approximation. See, for example, the books by Lorentz 104 and by DeVore and Lorentz 44. From (1.1) one can deduce in a standard fashion that if there is a sequence $\left(f_{n}\right)_{n=1}^{\infty}$ of exponential sums with $f_{n} \in E_{n}$ that approximates $f$ on an interval $[a, b]$ uniformly with errors

$$
\left\|f-f_{n}\right\|_{[a, b]}=O\left(n^{-m}(\log n)^{-2}\right), \quad n=2,3, \ldots,
$$

where $m \in \mathbb{N}$ is a fixed integer, then $f$ is $m$ times continuously differentiable on $(a, b)$. Let $\mathcal{P}_{n}$ be the collection of all polynomials of degree at most $n$ with real coefficients. Inequality (1.1) can be extended to $E_{n}$ and replaced by

$$
\widetilde{E}_{n}:=\left\{f: f(t)=a_{0}+\sum_{j=1}^{N} P_{m_{j}}(t) e^{\lambda_{j} t}, a_{0}, \lambda_{j} \in \mathbb{R}, \quad P_{m_{j}} \in \mathcal{P}_{m_{j}}, \quad \sum_{j=1}^{N}\left(m_{j}+1\right) \leq n\right\} .
$$

In fact, it is well known that $\widetilde{E}_{n}$ is the uniform closure of $E_{n}$ on any finite subinterval of the real number line; see Theorem 2.3 on page 173 of [41], for instance. For a function $f$ defined on a set $A$, let

$$
\|f\|_{A}:=\|f\|_{L_{\infty} A}:=\|f\|_{L_{\infty}(A)}:=\sup \{|f(x)|: x \in A\},
$$

and let

$$
\|f\|_{L_{p} A}:=\|f\|_{L_{p}(A)}:=\left(\int_{A}|f(x)|^{p} d x\right)^{1 / p}, \quad p>0,
$$

whenever the Lebesgue integral exists. In 65] we focused on the classes

$$
\begin{aligned}
G_{n} & :=\left\{f: f(t)=\sum_{j=1}^{n} a_{j} e^{-\left(t-\lambda_{j}\right)^{2}}, a_{j}, \lambda_{j} \in \mathbb{R}\right\}, \\
\widetilde{G}_{n}:=\{f: f(t) & =\sum_{j=1}^{N} P_{m_{j}}(t) e^{-\left(t-\lambda_{j}\right)^{2}}, \\
\lambda_{j} & \left.\in \mathbb{R}, P_{m_{j}} \in \mathcal{P}_{m_{j}}, \sum_{j=1}^{N}\left(m_{j}+1\right) \leq n\right\},
\end{aligned}
$$

and

$$
\begin{aligned}
\widetilde{G}_{n}^{*}:=\{f: f(t) & =\sum_{j=1}^{N} P_{m_{j}}(t) e^{-\left(t-\lambda_{j}\right)^{2}}, \\
\lambda_{j} & \left.\in\left[-n^{1 / 2}, n^{1 / 2}\right], \quad P_{m_{j}} \in \mathcal{P}_{m_{j}}, \sum_{j=1}^{N}\left(m_{j}+1\right) \leq n\right\} .
\end{aligned}
$$

Note that $\widetilde{G}_{n}$ is the uniform closure of $G_{n}$ on any finite subinterval of the real number line. Let $W(t):=\exp \left(-t^{2}\right)$. Combining Corollaries 1.5 and 1.8 in 45] and recalling that for the weight $W$ the Mhaskar-Rachmanov-Saff number $a_{n}$ defined by (1.4) in [45] satisfies $a_{n} \leq c_{1} n^{1 / 2}$ with a constant $c_{1}$ independent of $n$, we obtain

$$
\inf _{P \in \mathcal{P}_{n}}\|(P-g) W\|_{L_{q}(\mathbb{R})} \leq c_{2} n^{-m / 2}\left\|g^{(m)} W\right\|_{L_{q}(\mathbb{R})}
$$

with a constant $c_{2}$ independent of $n$, for every $g$ for which the norm on the right-hand side is finite for some $m \in \mathbb{N}$ and $q \in[1, \infty]$. As a consequence,

$$
\inf _{f \in \widetilde{G}_{n}^{*}}\|f-g W\|_{L_{q}(\mathbb{R})} \leq c_{3} n^{-m / 2} \sum_{k=0}^{m}\left\|(1+|t|)^{m-k}(g W)^{(k)}(t)\right\|_{L_{q}(\mathbb{R})}
$$


with a constant $c_{3}$ independent of $n$ for every $g$ for which the norms on the right-hand side are finite for each $k=0,1, \ldots, m$ with some $q \in[1, \infty]$. Replacing $g W$ by $g$, we conclude that

$$
\inf _{f \in \widetilde{G}_{n}^{*}}\|f-g\|_{L_{q}(\mathbb{R})} \leq c_{3} n^{-m / 2} \sum_{k=0}^{m}\left\|(1+|t|)^{m-k} g^{(k)}(t)\right\|_{L_{q}(\mathbb{R})}
$$

with a constant $c_{3}$ independent of $n$ for every $g$ for which the norms on the right-hand side are finite for each $k=0,1, \ldots, m$ with some $q \in[1, \infty]$. In [65] we proved the following results.

Theorem 1.1. There is an absolute constant $c_{4}$ such that

$$
\left|U_{n}^{\prime}(0)\right| \leq c_{4} n^{1 / 2}\left\|U_{n}\right\|_{\mathbb{R}}
$$

for all $U_{n}$ of the form $U_{n}=P_{n} Q_{n}$ with $P_{n} \in \widetilde{G}_{n}$ and an even $Q_{n} \in \mathcal{P}_{n}$. As a consequence,

$$
\left\|P_{n}^{\prime}\right\|_{\mathbb{R}} \leq c_{4} n^{1 / 2}\left\|P_{n}\right\|_{\mathbb{R}}
$$

for all $P_{n} \in \widetilde{G}_{n}$.

We remark that a closer look at the proof shows that $c_{4}=5$ in the above theorem is an appropriate choice.

Theorem 1.2. There is an absolute constant $c_{5}$ such that

$$
\left\|U_{n}^{\prime}\right\|_{L_{q}(\mathbb{R})} \leq c_{5}^{1+1 / q} n^{1 / 2}\left\|U_{n}\right\|_{L_{q}(\mathbb{R})}
$$

for all $U_{n} \in \widetilde{G}_{n}$ and $q \in(0, \infty)$.

Theorem 1.3. There is an absolute constant $c_{6}$ such that

$$
\left\|U_{n}^{(m)}\right\|_{L_{q}(\mathbb{R})} \leq\left(c_{6}^{1+1 / q} n m\right)^{m / 2}\left\|U_{n}\right\|_{L_{q}(\mathbb{R})}
$$

for all $U_{n} \in \widetilde{G}_{n}, q \in(0, \infty]$, and $m=1,2, \ldots$.

We remark that a closer look at the proofs shows that $c_{5}=180 \pi$ in Theorem 1.2 and $c_{6}=180 \pi$ in Theorem 1.3 are appropriate choices.

Our next theorem may be viewed as a slightly weaker version of the right inverse theorem of approximation that can be coupled with the direct theorem of approximation formulated in (1.2).

Theorem 1.4. Suppose $q \in[1, \infty], m$ is a positive integer, $\varepsilon>0$, and $f$ is a function defined on $\mathbb{R}$. Suppose also that

$$
\inf _{f_{n} \in \widetilde{G}_{n}}\left\|f_{n}-f\right\|_{L_{q}(\mathbb{R})} \leq c_{7} n^{-m / 2}(\log n)^{-1-\varepsilon}, \quad n=2,3, \ldots,
$$

with a constant $c_{7}$ independent of $n$. Then $f$ is $m$ times differentiable almost everywhere on $\mathbb{R}$. Also, if

$$
\inf _{f_{n} \in \widetilde{G}_{n}^{*}}\left\|f_{n}-f\right\|_{L_{q}(\mathbb{R})}=c_{7} n^{-m / 2}(\log n)^{-1-\varepsilon}, \quad n=2,3, \ldots,
$$

with a constant $c_{7}$ independent of $n$, then, in addition to the fact that $f$ is $m$ times differentiable almost everywhere on $\mathbb{R}$, we also have

$$
\left\|(1+|t|)^{m-j} f^{(j)}(t)\right\|_{L_{q}(\mathbb{R})}<\infty, \quad j=0,1, \ldots, m .
$$

Theorem 1.5. There is an absolute constant $c_{8}$ such that

$$
\left\|U_{n}^{\prime}\right\|_{L_{q}[y-\delta / 2, y+\delta / 2]} \leq c_{8}^{1+1 / q}\left(\frac{n}{\delta}\right)\left\|U_{n}\right\|_{L_{q}[y-\delta, y+\delta]}
$$

for all $U_{n} \in \widetilde{G}_{n}, q \in(0, \infty], y \in \mathbb{R}$, and $\delta \in\left(0, n^{1 / 2}\right]$. 
A multidimensional analog $G_{\mathbf{m}}$ of the class $G_{n}$ is introduced and studied briefly in $\S 7$. An element of $G_{\mathbf{m}}$ is called a Gaussian network of $N$ neurons. In this context H. Mhaskar [110] writes: "Professor Ward at Texas A\&M University has pointed out that our results implicitly contain an inequality, known as Bernstein inequality, in terms of the number of neurons, under some conditions on the minimal separation. Professor Erdélyi at Texas A\&M University has kindly sent us a manuscript in preparation, where he proves this inequality purely in terms of the number of neurons, with no further conditions. This inequality leads to the converse theorems in terms of the number of neurons, matching our direct theorem in this theory. Our direct theorem in 109 is sharp in the sense of $n$-widths. However, the converse theorem applies to individual functions rather than a class of functions. In particular, it appears that even if the cost of approximation is measured in terms of the number of neurons, if the degrees of approximation of a particular function by Gaussian networks decay polynomially, then a linear operator will yield the same order of magnitude in the error in approximating this function. We find this astonishing, since many people have told us, based on numerical experiments, that one can achieve a better degree of approximation by nonlinear procedures by stacking the centers near the bad points of the target functions". (The concept of width was introduced and examined thoroughly in Chapter 13 of [105].)

\section{§2. RemeZ-Type inequalities for EXPONENTial sums}

The classical Remez inequality [122] states that if $p$ is a polynomial of degree at most $n, s \in(0,2)$, and

then

$$
m(\{x \in[-1,1]:|p(x)| \leq 1\}) \geq 2-s
$$

$$
\|p\|_{[-1,1]} \leq T_{n}\left(\frac{2+s}{2-s}\right)
$$

where $T_{n}$, defined by

$$
T_{n}(x):=\cos (n \arccos x), \quad x \in[-1,1],
$$

is the Chebyshev polynomial of degree $n$. This inequality is sharp, and

$$
T_{n}\left(\frac{2+s}{2-s}\right) \leq \exp \left(\min \left\{5 n s^{1 / 2}, 2 n^{2} s\right\}\right), \quad s \in(0,1] .
$$

Remez-type inequalities turn out to be very useful in various problems of approximation theory. See, for example, Remez [122, Borwein and Erdélyi [17, 18, 25], and [27, Erdélyi [46, 51, and [52, Erdélyi and Nevai [75, Freud [83, p. 122], and Lorentz, Golitschek, and Makovoz [105]. Lorentz liked my essentially sharp Remez-type inequality for trigonometric polynomials [51] and he stated it in his book [105, p. 77] correctly.

Theorem 2.1. If $p$ is a trigonometric polynomial of degree at most $n, s \in(0, \pi / 2]$, and

$$
m(\{t \in[-\pi, \pi):|p(t)| \leq 1\}) \geq 2 \pi-s,
$$

then

$$
\|p\|_{[-\pi, \pi]} \leq \exp (\text { cns })
$$

with an absolute constant $c>0(c=2$ is a suitable choice $)$.

He writes: "The proof of this trigonometric Remez-type inequality needs new ideas, and is far from being a copy of the methods working in the algebraic case." My memory is that at one point Lorentz sent me an approach that seemed to reduce the proof of my Remez-type inequality for trigonometric polynomials to the Remez inequality for algebraic polynomials. The mistake in it was so subtle that at first I approved it. Later, when I observed a gap in it, Lorentz decided he would just state the result without proof. 
I think even today only my paper [51] contains the trigonometric Remez inequality with a correct proof.

Another remarkable result in [51] is the following essentially pointwise Remez-type inequality for algebraic polynomials.

Theorem 2.2. If $p$ is an algebraic polynomial of degree at most $n, s \in(0,1], y \in(-1,1)$, and

then

$$
m(\{x \in[-1,1]:|p(x)| \leq 1\}) \geq 2-s,
$$

$$
|p(y)| \leq \exp \left(\operatorname{cn} \min \left\{\frac{s}{\sqrt{1-y^{2}}}, \sqrt{s}\right\}\right)
$$

with an absolute constant $c>0$.

In fact, both Theorems 2.1 and 2.2 easily extend to the classes $\operatorname{GTP}_{N}$ and $\operatorname{GAP}_{N}$, respectively, of generalized (trigonometric) polynomials. These classes are introduced in $\S 10$, where inequalities for generalized polynomials from the classes $\operatorname{GTP}_{N}$ and $\mathrm{GAP}_{N}$ are discussed.

In [27], we proved the following result.

Theorem 2.3 (Remez-type inequality for $E_{n}$ at 0$)$. Let $s \in\left(0, \frac{1}{2}\right]$. There are absolute constants $c_{2}>0$ and $c_{3}>0$ such that

$$
\frac{1}{2} \exp \left(c_{2} n s\right) \leq \sup _{f}|f(0)| \leq \exp \left(c_{3} n s\right),
$$

where the supremum is taken for all $f \in E_{n}$ satisfying

$$
m(\{x \in[-1,1]:|f(x)| \leq 1\}) \geq 2-s .
$$

In fact, in [27, Theorem 2.2 was stated carelessly. The factor $1 / 2$ on the left-hand side was missing. More accurate estimates for the values of the Chebyshev polynomial appearing in the proof of the above result give the following more complete result.

Theorem 2.3* (Remez-type inequality for $E_{n}$ at 0$)$. Let $s \in\left(0, \frac{1}{2}\right]$. There are absolute constants $c_{2}>0$ and $c_{3}>0$ such that

$$
\exp \left(c_{2} \min \left\{n s,(n s)^{2}\right\}\right) \leq \sup _{f}|f(0)| \leq \exp \left(c_{3} \min \left\{n s,(n s)^{2}\right\}\right),
$$

where the supremum is taken for all $f \in E_{n}$ satisfying

$$
m(\{x \in[-1,1]:|f(x)| \leq 1\}) \geq 2-s .
$$

In [67, we established an essentially sharp Remez-type inequality for $G_{n}$ and $\widetilde{G}_{n}$. We also proved the right higher-dimensional analog of our main result.

Theorem 2.4 (Remez-type inequality for $\left.\widetilde{G}_{n}\right)$. Let $s \in(0, \infty)$ and $n \geq 9$. There is an absolute constant $c_{1}>0$ such that

$$
\exp \left(c_{1}\left(\min \left\{n^{1 / 2} s, n s^{2}\right\}+s^{2}\right)\right) \leq \sup _{f}\|f\|_{\mathbb{R}} \leq \exp \left(80\left(\min \left\{n^{1 / 2} s, n s^{2}\right\}+s^{2}\right)\right),
$$

where the supremum is taken for all $f \in \widetilde{G}_{n}$ satisfying

$$
m(\{t \in \mathbb{R}:|f(t)| \geq 1\}) \leq s .
$$

Important results of Turán [137] are based on the following observations. Let

$$
g(\nu):=\sum_{j=1}^{n} b_{j} z_{j}^{\nu}, \quad b_{j}, z_{j} \in \mathbb{C} .
$$


Suppose

$$
\min _{1 \leq j \leq n}\left|z_{j}\right| \geq 1, \quad j=1,2, \ldots, n
$$

Then

$$
\max _{\nu=m+1, \ldots, m+n}|g(\nu)| \geq\left(\frac{n}{2 e(m+n)}\right)^{n}\left|b_{1}+b_{2}+\cdots+b_{n}\right|
$$

for every positive integer $m$.

A consequence of the preceding is the famous Turán lemma: if

$$
f(t):=\sum_{j=1}^{n} b_{j} e^{\lambda_{j} t}, \quad b_{j}, \lambda_{j} \in \mathbb{C},
$$

and

$$
\min _{1 \leq j \leq n} \operatorname{Re}\left(\lambda_{j}\right) \geq 0
$$

then

$$
|f(0)| \leq\left(\frac{2 e(a+d)}{d}\right)^{n}\|f\|_{[a, a+d]}
$$

for every $a>0$ and $d>0$.

Another consequence of this is the fact that if

$$
p(z):=\sum_{j=1}^{n} b_{j} z^{\lambda_{j}}, \quad b_{j} \in \mathbb{C}, \quad \lambda_{j} \in \mathbb{R}, \quad z=e^{i \theta},
$$

then

$$
\max _{|z|=1}|p(z)| \leq\left(\frac{4 e \pi}{\delta}\right)^{n} \max _{\substack{|z|=1 \\ \alpha \leq \arg (z) \leq \alpha+\delta}}|p(z)|
$$

for every $0 \leq \alpha<\alpha+\delta \leq 2 \pi$.

Turán's inequalities above and their variants play a central role in the book of Turán [137, where many applications are also presented. The main point in these inequalities is that the exponent on the right-hand side is only the number of terms $n$, and so it is independent of the numbers $\lambda_{j}$. An inequality of the type

$$
\max _{|z|=1}|p(z)| \leq c(\delta)^{\lambda_{n}} \max _{\substack{|z|=1 \\ \alpha \leq \arg (z) \leq \alpha+\delta}}|p(z)|,
$$

where $0 \leq \lambda_{1}<\lambda_{2}<\cdots<\lambda_{n}$ are integers and $c(\delta)$ depends only on $\delta$, could be obtained by a simple direct argument, but it is much less useful than Turán's inequality. F. Nazarov has a seminal paper [113] devoted to Turán-type inequalities for exponential sums and their applications to various uniqueness theorems in harmonic analysis of the uncertainty principle type. The author derived an estimate for the maximum modulus of exponential sums of the form (2.1) on an interval $I \subset \mathbb{R}$ in terms of its maximum modulus on a measurable set $E \subset I$ of positive Lebesgue measure:

$$
\sup _{t \in I}|f(t)| \leq e^{\max \left|\operatorname{Re} \lambda_{k}\right| m(I)}\left(\frac{A m(I)}{m(E)}\right)^{n-1} \sup _{t \in E}|f(t)|,
$$

where $A$ is an absolute constant. 


\section{§3. LORENTZ DEGREE OF POLYNOMIALS}

In 1915, S. N. Bernstein [8] observed that any polynomial $p$ having no zeros in the interval $(-1,1)$ can be written in the form

$$
p(x)=\sum_{j=0}^{d} a_{j}(1-x)^{j}(1+x)^{d-j}
$$

with all $a_{j} \geq 0$. Hausdorff claimed this result independently, and the Pólya-Szegö book attributes it to him [87, pp. 98-99]. The smallest natural number $d$ for which such a representation holds is called the Lorentz degree of $p$ and it is denoted by $d(p)$. The Lorentz degree was named after George Lorentz, who established essentially sharp Markov- and Bernstein-type inequalities on $[-1,1]$ for polynomials of Lorentz degree $d$, and one of his students, J. T. Scheick [127], contributed to the topic substantially. The Lorentz degree $d(p)$ can be much larger than the usual degree of the polynomial $p$. Nevertheless, in [48] and 73 , essentially sharp upper and lower bounds were given for classes of polynomials of usual degree $n$ having no zeros in ellipses with axes $[-1,1]$ and $[-\varepsilon i, \varepsilon i], \varepsilon \in(0,1]$. As a by-product, the proofs of Lorentz's Markov- and Bernstein-type inequalities [102] were shortened so that they fit to print in his book [44, p. 115] with DeVore.

To formulate our main theorem from [48, we need some notation. Let $\varphi$ be a positive continuous function defined on $(-1,1)$, and let

$$
D(\varphi):=\{z=x+i y:|y|<\varphi(x),|x|<1\}
$$

denote the domain of the complex plane determined by it. We introduce

$$
L_{n}(\varphi):=\left\{p \in \mathcal{P}_{n}: p(z) \neq 0, \quad z \in D(\varphi)\right\}
$$

and

$$
d_{n}(\varphi):=\sup _{p \in L_{n}(\varphi)} d(p)
$$

Sharpening a result in [73, Theorem 3], we proved the following in [48].

Theorem 3.1. If

$$
1 \geq \varepsilon:=\inf _{x \in(-1,1)} \frac{\varphi(x)}{\sqrt{1-x^{2}}}>0
$$

then

where $0<c_{1}<c_{2}$ are absolute constants.

$$
\frac{c_{1} n}{\varepsilon^{2}} \leq d_{n}(\varphi) \leq \frac{c_{2} n}{\varepsilon^{2}}
$$

Let

$$
p(x):=(1-x)^{2}-2(1-x)(1+x)+4(1+x)^{2} \text { and } q(x):=(1+x)+\frac{1}{2}(1-x) .
$$

Then $d(p)=4, d(q)=1$, and $d(p q)=3$. As far as I know, the following two questions raised in [48] are still open. Is it true that $d(p q) \geq \min \{d(p), d(q)\}$ for any polynomials $p$ and $q$ ? Is it true that $d(p q) \geq|d(p)-d(q)|$ for any polynomials $p$ and $q$ ?

\section{§4. MARKOV- AND Bernstein-TyPe Inequalities FOR POLYNOMIALS WITH CONSTRAINTS}

The Markov-Bernstein inequality asserts that

$$
\left|p^{\prime}(x)\right| \leq \min \left\{\frac{n}{\sqrt{1-x^{2}}}, n^{2}\right\}\|p\|_{[-1,1]}, \quad x \in(-1,1),
$$

for every polynomial of degree at most $n$ with complex coefficients. Here and in what follows, $\|p\|_{A}:=\sup _{y \in A}|p(y)|$. Throughout his life Erdös showed a particular interest 
in inequalities for constrained polynomials. In a short paper in 1940, Erdős [78 found a class of restricted polynomials for which the Markov factor $n^{2}$ improves to $\mathrm{cn}$. He proved that there is an absolute constant $c$ such that

$$
\left|p^{\prime}(x)\right| \leq \min \left\{\frac{c \sqrt{n}}{\left(1-x^{2}\right)^{2}}, \frac{e n}{2}\right\}\|p\|_{[-1,1]}, \quad x \in(-1,1),
$$

for every polynomial $p$ of degree at most $n$ that has all its zeros in $\mathbb{R} \backslash(-1,1)$. This result motivated a number of people to study Markov- and Bernstein-type inequalities for polynomials with restricted zeros and under some other constraints. Generalizations of the above Markov-Bernstein-type inequality of Erdős were extended later in many directions.

Let $\mathcal{P}_{n, k}^{c}$ denote the set of all polynomials of degree at most $n$ with complex coefficients and with at most $k(0 \leq k \leq n)$ zeros in the open unit disk. Let $\mathcal{P}_{n, k}$ denote the set of all polynomials of degree at most $n$ with real coefficients and with at most $k(0 \leq k \leq n)$ zeros in the open unit disk. Associated with $0 \leq k \leq n$ and $x \in(-1,1)$, let

$$
B_{n, k, x}^{*}:=\max \left\{\sqrt{\frac{n(k+1)}{1-x^{2}}}, n \log \left(\frac{e}{1-x^{2}}\right)\right\}, \quad B_{n, k, x}:=\sqrt{\frac{n(k+1)}{1-x^{2}}},
$$

and

$$
M_{n, k}^{*}:=\max \{n(k+1), \quad n \log n\}, \quad M_{n, k}:=n(k+1) .
$$

In [53] and [54] it was shown that

$$
c_{1} \min \left\{B_{n, k, x}^{*}, M_{n, k}^{*}\right\} \leq \sup _{p \in \mathcal{P}_{n, k}^{c}} \frac{\left|p^{\prime}(x)\right|}{\|p\|_{[-1,1]}} \leq c_{2} \min \left\{B_{n, k, x}^{*}, M_{n, k}^{*}\right\}
$$

for every $x \in(-1,1)$, where $c_{1}>0$ and $c_{2}>0$ are absolute constants. This result should be compared with the inequalities

$$
c_{3} \min \left\{B_{n, k, x}, M_{n, k}\right\} \leq \sup _{p \in \mathcal{P}_{n, k}} \frac{\left|p^{\prime}(x)\right|}{\|p\|_{[-1,1]}} \leq c_{4} \min \left\{B_{n, k, x}, M_{n, k}\right\}
$$

for every $x \in(-1,1)$, where $c_{3}>0$ and $c_{4}>0$ are absolute constants. The upper bound of this second result may be found in [14, and it may be surprising that there is a significant difference between the real and complex cases as far as Markov-Bernstein-type inequalities are concerned. The lower bound of the second result was proved in [53. It is the final piece of a long series of papers on this topic by a number of authors starting with Erdös in 1940. In addition, in [15], we established the right Markov-Bernstein-type inequalities for the classes $\mathcal{P}_{n, k}$ in $L_{p}[-1,1], p>0$.

Let $\mathcal{P}_{n}^{c}(r)$ be the set of all polynomials of degree at most $n$ with complex coefficients and with no zeros in the union of open disks with diameters $[-1,-1+2 r]$ and $[1-2 r, 1]$ $(0<r \leq 1)$. Let $\mathcal{P}_{n}(r)$ be the set of all polynomials of degree at most $n$ with real coefficients and with no zeros in the union of open disks with diameters $[-1,-1+2 r]$ and $[1-2 r, 1](0<r \leq 1)$.

Essentially sharp Markov-type inequalities for $\mathcal{P}_{n}^{c}(r)$ and $\mathcal{P}_{n}(r)$ on $[-1,1]$ were established in [53] and [47]. In [53], we showed that

$$
c_{1} \min \left\{\frac{n \log (e+n \sqrt{r})}{\sqrt{r}}, n^{2}\right\} \leq \sup _{0 \neq p \in \mathcal{P}_{n}^{c}(r)} \frac{\left\|p^{\prime}\right\|_{[-1,1]}}{\|p\|_{[-1,1]}} \leq c_{2} \min \left\{\frac{n \log (e+n \sqrt{r})}{\sqrt{r}}, n^{2}\right\}
$$

for every $0<r \leq 1$ with absolute constants $c_{1}>0$ and $c_{2}>0$. This result should be compared with the inequalities

$$
c_{3} \min \left\{\frac{n}{\sqrt{r}}, n^{2}\right\} \leq \sup _{0 \neq p \in \mathcal{P}_{n}(r)} \frac{\left\|p^{\prime}\right\|_{[-1,1]}}{\|p\|_{[-1,1]}} \leq c_{4} \min \left\{\frac{n}{\sqrt{r}}, n^{2}\right\}, \quad 0<r \leq 1,
$$


where $c_{3}>0$ and $c_{4}>0$ are absolute constants. See [4].

Lorentz included a more general version of the above result for higher derivatives, allowing $k(0 \leq k \leq n)$ zeros in the union of open disks with diameters $[-1,-1+2 r]$ and $[1-2 r, 1]$, respectively $(0<r \leq 1)$. In his book [105, pp. 64-73], he followed the proof in [47, where the idea of "Lorentz representation" turned out to be crucial. Note that induction does not work here due to the lack of a Gauss-Lucas theorem.

Let $K_{\alpha}$ be the open diamond of the complex plane with diagonals [-1,1] and [-ia, ia] such that the angle between $[i a, 1]$ and $[1,-i a]$ is $\alpha \pi$. In [85], Halász proved that there are constants $c_{1}>0$ and $c_{2}>0$ depending only on $\alpha$ such that

$$
c_{1} n^{2-\alpha} \leq \sup _{p} \frac{\left|p^{\prime}(1)\right|}{\|p\|_{[-1,1]}} \leq \sup _{p} \frac{\left\|p^{\prime}\right\|_{[-1,1]}}{\|p\|_{[-1,1]}} \leq c_{2} n^{2-\alpha},
$$

where the supremum is taken for all polynomials $p$ of degree at most $n$ (with either real or complex coefficients) having no zeros in $K_{\alpha}$.

Erdős had many questions and results about polynomials with restricted coefficients. Let $\mathcal{F}_{n}$ denote the set of polynomials of degree at most $n$ with coefficients from $\{-1,0,1\}$. Let $\mathcal{G}_{n}$ be the collection of polynomials $p$ of the form

$$
p(x)=\sum_{j=m}^{n} a_{j} x^{j}, \quad\left|a_{m}\right|=1, \quad\left|a_{j}\right| \leq 1,
$$

where $m$ is an unspecified nonnegative integer not greater than $n$. In [28] and [30, we established the right Markov-type inequalities for the classes $\mathcal{F}_{n}$ and $\mathcal{G}_{n}$ on $[0,1]$. Namely, there are absolute constants $c_{1}>0$ and $c_{2}>0$ such that

$$
c_{1} n \log (n+1) \leq \max _{0 \neq p \in \mathcal{F}_{n}} \frac{\left\|p^{\prime}\right\|_{[0,1]}}{\|p\|_{[0,1]}} \leq c_{2} n \log (n+1)
$$

and

$$
c_{1} n^{3 / 2} \leq \max _{0 \neq p \in \mathcal{G}_{n}} \frac{\left\|p^{\prime}\right\|_{[0,1]}}{\|p\|_{[0,1]}} \leq c_{2} n^{3 / 2} .
$$

Observe that the right Markov factor for $\mathcal{G}_{n}$ is much larger than the right Markov factor for $\mathcal{F}_{n}$. We also show that there are absolute constants $c_{1}>0$ and $c_{2}>0$ such that

$$
c_{1} n \log (n+1) \leq \max _{0 \neq p \in \mathcal{L}_{n}} \frac{\left\|p^{\prime}\right\|_{[0,1]}}{\|p\|_{[0,1]}} \leq c_{2} n \log (n+1),
$$

where $\mathcal{L}_{n}$ denotes the set of polynomials of degree at most $n$ with coefficients from $\{-1,1\}$.

For polynomials

$$
p \in \mathcal{F}:=\bigcup_{n=0}^{\infty} \mathcal{F}_{n} \quad \text { with } \quad|p(0)|=1
$$

and for $y \in[0,1)$, the Bernstein-type inequality

$$
\frac{c_{1} \log \left(\frac{2}{1-y}\right)}{1-y} \leq \max _{\substack{p \in \mathcal{F} \\|p(0)|=1}} \frac{\left\|p^{\prime}\right\|_{[0, y]}}{\|p\|_{[0,1]}} \leq \frac{c_{2} \log \left(\frac{2}{1-y}\right)}{1-y}
$$

was also proved in [30] with absolute constants $c_{1}>0$ and $c_{2}>0$.

Let $\mathcal{P}_{n}^{m}$ be the collection of all polynomials of degree at most $n$ with real coefficients that have at most $m$ distinct complex zeros. In [6], we proved that

$$
\max _{x \in[0,1]}\left|P^{\prime}(x)\right| \leq 32 \cdot 8^{m} n \max _{x \in[0,1]}|P(x)|
$$

for every $P \in \mathcal{P}_{n}^{m}$. This is far from what we expect. We conjecture that the Markov factor $32 \cdot 8^{m} n$ above may be replaced by $c m n$ with an absolute constant $c>0$. We 
are not able to prove this conjecture at the moment. However, we think that our result above gives the best-known Markov-type inequality for $\mathcal{P}_{n}^{m}$ on a finite interval when $m \leq c \log n$.

For continuous functions $p$ defined on the complex unit circle, and for $q \in(0, \infty)$, we define

We also define

$$
\|p\|_{q}:=\left(\int_{0}^{2 \pi}\left|p\left(e^{i t}\right)\right|^{q} d t\right)^{1 / q} .
$$

$$
\|p\|_{\infty}:=\lim _{q \rightarrow \infty}\|p\|_{q}=\max _{t \in[0,2 \pi]}\left|p\left(e^{i t}\right)\right| .
$$

Based on the ideas of F. Nazarov, Queffélec and Saffari [125] showed that

$$
\sup _{p \in \mathcal{L}_{n}} \frac{\left\|p^{\prime}\right\|_{q}}{\|p\|_{q}}=\gamma_{n, q} n, \quad \lim _{n \rightarrow \infty} \gamma_{n, q}=1
$$

for every $q \in(0, \infty], q \neq 2$ (when $q=2, \lim _{n \rightarrow \infty} \gamma_{n, q}=3^{-1 / 2}$ by the Parseval formula). This shows that Bernstein's classical inequality (extended by Arestov [2] for all $q \in(0, \infty]$ ) stating that

$$
\left\|p^{\prime}\right\|_{q} \leq n\|p\|_{q}
$$

for all polynomials of degree at most $n$ with complex coefficients, cannot be essentially improved for the class $\mathcal{L}_{n}$, except for the trivial $q=2$ case.

Let $\mathrm{SR}_{n}^{c}$ denote the set of all self-reciprocal polynomials $p_{n} \in \mathcal{P}_{n}^{c}$ satisfying

$$
p_{n}(z)=z^{n} p_{n}\left(z^{-1}\right) .
$$

Let $\mathrm{SR}_{n}$ denote the set of all real self-reciprocal polynomials of degree at most $n$, that is, $\mathrm{SR}_{n}:=\mathrm{SR}_{n}^{c} \cap \mathcal{P}_{n}$. For a polynomial $p_{n} \in \mathcal{P}_{n}^{c}$ of the form

$$
p_{n}(z)=\sum_{j=0}^{n} a_{j} z^{j}, \quad a_{j} \in \mathbb{C}
$$

$p_{n} \in \mathrm{SR}_{n}^{c}$ if and only if

$$
a_{j}=a_{n-j}, \quad j=0,1, \ldots, n .
$$

Let $\mathrm{ASR}_{n}^{c}$ denote the set of all anti-self-reciprocal polynomials $p_{n} \in \mathcal{P}_{n}^{c}$ satisfying

$$
p_{n}(z)=-z^{n} p_{n}\left(z^{-1}\right) .
$$

Let $\mathrm{ASR}_{n}$ denote the set of all real anti-self-reciprocal polynomials, that is, $\mathrm{ASR}_{n}:=$ $\mathrm{ASR}_{n}^{c} \cap \mathcal{P}_{n}$. For a polynomial $p_{n} \in \mathcal{P}_{n}^{c}$ of the form (4.1), $p_{n} \in \mathrm{ASR}_{n}^{c}$ if and only if

$$
a_{j}=-a_{n-j}, \quad j=0,1, \ldots, n .
$$

Every $p_{n} \in \mathrm{SR}_{n}^{c}$ and $p_{n} \in \mathrm{ASR}_{n}^{c}$ satisfies the growth condition

$$
\left|p_{n}(x)\right| \leq\left(1+|x|^{n}\right)\left\|p_{n}\right\|_{[-1,1]}, \quad x \in[-1,1] .
$$

The Markov-type (uniform) part of the following inequality is due to Kroó and Szabados [101. For the Bernstein-type (pointwise) part, see [17.

Theorem 4.1. There is an absolute constant $c_{1}>0$ such that

$$
\left|p_{n}^{\prime}(x)\right| \leq c_{1} n \min \left\{\log n, \log \left(\frac{e}{1-x^{2}}\right)\right\}\left\|p_{n}\right\|_{[-1,1]}
$$

for every $x \in(-1,1)$ and for every polynomial $p_{n} \in \mathcal{P}_{n}^{c}$ satisfying the growth condition (4.2), in particular for every $p_{n} \in \mathrm{SR}_{n}^{c}$ and for every $p_{n} \in \operatorname{ASR}_{n}^{c}(n \geq 2)$. 
In [17, it was shown that the above result is sharp for the classes $\mathrm{SR}_{n}$ and $\mathrm{ASR}_{n}$; that is, there are absolute constants $c_{1}>0$ and $c_{2}>0$ such that

$$
c_{1} n \min \left\{\log n, \log \left(\frac{e}{1-x^{2}}\right)\right\} \leq \sup _{p_{n}} \frac{\left|p^{\prime}(x)\right|}{\left\|p_{n}\right\|_{[-1,1]}} \leq c_{2} n \min \left\{\log n, \log \left(\frac{e}{1-x^{2}}\right)\right\},
$$

where the supremum is taken either for all $0 \neq p_{n} \in \mathrm{SR}_{n}$ or for all $0 \neq p_{n} \in \mathrm{ASR}_{n}$ $(n \geq 2)$. Associated with a polynomial $p_{n} \in \mathcal{P}_{n}^{c}$ of the form (4.1), we define the polynomial

$$
p_{n}^{*}(z)=\sum_{j=0}^{n} \bar{a}_{n-j} z^{j}
$$

Let $D$ and $\partial D$ denote the open unit disk and the unit circle, respectively, of the complex plane. It is well known and was proved in [106, p. 689] that

$$
\max _{z \in \partial D}\left(\left|p_{n}^{\prime}(z)\right|+\left|p_{n}^{* \prime}(z)\right|\right)=n \max _{z \in \partial D}\left|p_{n}(z)\right| .
$$

In particular, if $p_{n} \in \mathcal{P}_{n}^{c}$ is conjugate reciprocal (satisfying $p_{n}=p_{n}^{*}$ ), then

$$
\max _{z \in \partial D}\left|p_{n}^{\prime}(z)\right| \leq \frac{n}{2} \max _{z \in \partial D}\left|p_{n}(z)\right| \text {. }
$$

In [120], the inequality

$$
\max _{z \in \partial D}\left|p_{n}^{\prime}(z)\right| \leq(n-1 / 4) \max _{z \in \partial D}\left|p_{n}(z)\right|
$$

was stated for all $p_{n} \in \mathrm{SR}_{n}^{c}$. In this inequality, in general, the Bernstein factor $(n-1 / 4)$ cannot be replaced by anything better than $(n-1)$, as the following example shows. Let $P \in \mathcal{P}_{4 n+4}^{c}$ be defined by

$$
P\left(e^{i t}\right)=(\cos ((2 n+1) t)+i(\sin ((2 n+1) t) \sin t)) e^{i(2 n+2) t}, \quad t \in \mathbb{R} .
$$

Since $Q(t):=\cos ((2 n+1) t) \in \mathcal{T}_{2 n+1}$ and $R(t):=\sin ((2 n+1) t) \sin t \in \mathcal{T}_{2 n+2}$ are even (real) trigonometric polynomials, $P \in \mathcal{P}_{4 n+4}^{c}$ is a self-reciprocal polynomial. Obviously $\|P\|_{\partial D} \leq 1$ since

$$
\cos ^{2}((2 n+1) t)+\sin ^{2}((2 n+1) t) \sin ^{2} t \leq 1
$$

Also

$$
i P^{\prime}\left(e^{i \pi / 2}\right) e^{i \pi / 2}=(2 n+1)(-1)^{n+1} e^{i(2 n+2) \pi / 2}+(2 n+2)(-1)^{n+1} e^{(2 n+2) \pi / 2} ;
$$

hence

$$
\left\|P^{\prime}\right\|_{\partial D} \geq 4 n+3 \geq(4 n+3)\|P\|_{\partial D}
$$

\section{§5. MÜNTZ-TYPE THEOREMS}

Müntz's classical theorem [112] characterizes sequences $\Lambda:=\left(\lambda_{j}\right)_{j=0}^{\infty}$ with

$$
0=\lambda_{0}<\lambda_{1}<\lambda_{2}<\cdots
$$

for which the Müntz space $M(\Lambda):=\operatorname{span}\left\{x^{\lambda_{0}}, x^{\lambda_{1}}, \ldots\right\}$ is dense in $C[0,1]$. Here, $M(\Lambda)$ is the collection of all finite linear combinations of the functions $x^{\lambda_{0}}, x^{\lambda_{1}}, \ldots$ with real coefficients, and $C(A)$ is the space of all real-valued continuous functions on $A \subset[0, \infty)$ equipped with the uniform norm. If $A:=[a, b]$ is a finite closed interval, then the notation $C[a, b]:=C([a, b])$ is used.

Müntz's Theorem. Suppose $\Lambda:=\left(\lambda_{j}\right)_{j=0}^{\infty}$ is a sequence satisfying $0=\lambda_{0}<\lambda_{1}<\cdots$. Then $M(\Lambda)$ is dense in $C[0,1]$ if and only if

$$
\sum_{j=1}^{\infty} \frac{1}{\lambda_{j}}=\infty
$$


Extending a result of Clarkson and Erdös [42, in 25] we proved the right Müntz-type theorem on compact subsets of $[0, \infty)$ with positive Lebesgue measure.

Theorem 5.1. If $\Lambda:=\left(\lambda_{j}\right)_{j=0}^{\infty}$ is an increasing sequence of nonnegative real numbers with $\lambda_{0}=0$, and $A \subset[0, \infty)$ is a compact set with positive Lebesgue measure, then $M(\Lambda)$ is dense in $C(A)$ if and only if (5.1) holds. Moreover, if (5.1) does not hold, then every function in the $C[0,1]$-closure of $H(\Lambda):=\operatorname{span}\left\{1, x^{\lambda_{1}}, x^{\lambda_{2}}, \ldots\right\}$ can be represented as an analytic function on $\left\{z \in \mathbb{C} \backslash(-\infty, 0]:|z|<r_{A}\right\}$ restricted to $\left(0, r_{A}\right)$, where

$$
r_{A}:=\sup \{x \in A: m(A \cap[x, \infty)\}>0 .
$$

See also [18. This result had been expected by Erdős and others for a long time. Lorentz liked this then quite recent result too and stated it in his book [105. In fact, the key to the proof of Theorem 5.1 is the bounded Remez-type inequality for nondense Müntz spaces, the key result in [25] and [18].

Theorem 5.2. For every increasing sequence $\Lambda:=\left(\lambda_{j}\right)_{j=0}^{\infty}$ of nonnegative real numbers with $\lambda_{0}=0$ for which (5.1) does not hold, there is a constant $c$ depending only on $\Lambda$ and $s$ (and not on $A$ or the number of terms in $p$ ) so that

$$
\|p\|_{[0, \inf A]} \leq c\|p\|_{A}
$$

for every $p \in \operatorname{span}\left\{x^{\lambda_{0}}, x^{\lambda_{1}}, \ldots\right\}$ and for every $A \subset[0,1]$ of Lebesgue measure at least $s \in(0,1)$.

Extending earlier results of Müntz, Szász, Clarkson, Erdős, L. Schwartz, P. Borwein, Erdélyi, and Operstein, in [72] we proved the result below.

Theorem 5.3 ("Full Müntz theorem" in $L_{p}[0,1]$ for $\left.p \in(0, \infty)\right)$. Let $p \in(0, \infty)$. Suppose $\left(\lambda_{j}\right)_{j=1}^{\infty}$ is a sequence of distinct real numbers greater than $-(1 / p)$. Then $H(\Lambda)$ is dense in $L_{p}[0,1]$ if and only if

$$
\sum_{j=1}^{\infty} \frac{\lambda_{j}+(1 / p)}{\left(\lambda_{j}+(1 / p)\right)^{2}+1}=\infty .
$$

Moreover, if (5.2) does not hold, then every function in the $L_{p}[0,1]$-closure of $H(\Lambda)$ can be represented as an analytic function on $\{z \in \mathbb{C} \backslash(-\infty, 0]:|z|<1\}$ restricted to $(0,1)$.

In handling the nondense case, that is, the case where (5.2) does not hold, in [72] we needed to refer to [4].

In [72, the authors were not able to include the case of $p=\infty$ in their discussion. The right result for $p=\infty$ was proved in [61].

Theorem 5.4 ("Full Clarkson-Erdős-Schwartz theorem" in $C[0,1])$. Suppose $\left(\lambda_{j}\right)_{j=1}^{\infty}$ is a sequence of distinct positive numbers. Then $\operatorname{span}\left\{1, x^{\lambda_{1}}, x^{\lambda_{2}}, \ldots\right\}$ is dense in $C[0,1]$ if and only if

$$
\sum_{j=1}^{\infty} \frac{\lambda_{j}}{\lambda_{j}^{2}+1}=\infty .
$$

Moreover, if (5.3) does not hold, then every function in the $C[0,1]$-closure of $H(\Lambda)$ can be represented as an analytic function on $\{z \in \mathbb{C} \backslash(-\infty, 0]:|z|<1\}$ restricted to $(0,1)$.

This result improves an earlier result by P. Borwein and Erdélyi (see [20] and [17]) stating that if (5.1) does not hold, then every function in the $C[0,1]$-closure of $H(\Lambda)$ is in $C^{\infty}(0,1)$.

In [64], we presented the proof of the above "full Müntz theorem" in $L_{p}[0,1]$ for $p \in(0, \infty)$ by using more elementary textbook methods.

The following problems are still open. 
Problem 5.5. Characterize the compact sets $A \subset[0, \infty)$ for which "Müntz's theorem holds", that is, for which $\operatorname{span}\left\{x^{\lambda_{0}}, x^{\lambda_{1}}, \ldots\right\}$ is dense in $C(A)$ if and only if (5.2) holds.

Problem 5.6. Does Müntz's theorem hold on every compact set $A \subset[0, \infty)$ of positive logarithmic capacity?

Problem 5.7. Does Müntz's theorem hold on the Cantor ternary set?

Problem 5.8. Is there a compact set $A \subset[0, \infty)$ of Lebesgue measure 0 on which Müntz's theorem holds?

\section{§6. RemeZ-type inequalities ANd Newman's PROduCt PROBlem}

Let $\Lambda:=\left(\lambda_{j}\right)_{j=0}^{\infty}$ be a sequence of distinct real numbers. Let

$$
R(\Lambda):=\left\{\frac{p}{q}: p, q \in \operatorname{span}\left\{x^{\lambda_{0}}, x^{\lambda_{1}}, \ldots\right\}\right\} .
$$

A surprising result of Somorjai [131] and Bak and Newman [3] is the following.

Theorem 6.1. $R(\Lambda) \cap C[0,1]$ is always dense in $C[0,1]$.

So division has extra usefulness. Can multiplication have this extra utility? In [115, p. 51] Newman wrote: "Thus we have the very sane, if very prosaic, question: $\mathrm{P}(10.6)$ Are the functions $\left(\sum a_{i} x^{i^{2}}\right)\left(\sum a_{j} x^{j^{2}}\right)$ dense in $\mathrm{C}[0,1]$ ?". In a more general setting, let $\Lambda:=\left(\lambda_{j}\right)_{j=0}^{\infty}$ be a sequence of distinct nonnegative real numbers with $\lambda_{0}=0$. Let

$$
M^{k}(\Lambda):=\left\{\prod_{j=1}^{k} p_{j}: p_{j} \in \operatorname{span}\left\{x^{\lambda_{0}}, x^{\lambda_{1}}, \ldots\right\}\right\} .
$$

Suppose $k \geq 2$ and $\sum_{j=1}^{\infty} 1 / \lambda_{j}<\infty$. Can $M^{k}(\Lambda)$ be dense in $C[0,1]$ ? In [25] we solved this Newman product problem. In fact, we had observed before that it would be a reasonably simple consequence of our bounded Remez-type inequality, Theorem 5.2, for nondense Müntz spaces.

Theorem 6.2. If $\sum_{j=1}^{\infty} 1 / \lambda_{j}<\infty, k \geq 2$, and $A \subset[0, \infty)$ is a compact set of positive Lebesgue measure, then $M^{k}(\Lambda)$ is not dense in $C(A)$.

Remark 6.3. $M^{k}(\Lambda)$ is contained in (not equal to)

$$
\operatorname{span}\left\{x^{\lambda_{j_{1}}+\lambda_{j_{2}}+\cdots+\lambda_{j_{k}}}: \lambda_{j_{1}}, \lambda_{j_{2}}, \ldots, \lambda_{j_{k}} \in \Lambda\right\} .
$$

Example 6.4. Let $\Lambda:=\left(\lambda_{j}\right)_{j=0}^{\infty}$ be defined by

$$
\lambda_{i}:= \begin{cases}0, & j=0, \\ 2^{j-1}, & j=1,2, \ldots .\end{cases}
$$

Then

$$
\sum_{\lambda_{j_{1}, \lambda_{j_{2}}, \ldots, \lambda_{j_{k}} \in \Lambda}} \frac{1}{\lambda_{j_{1}}+\lambda_{j_{2}}+\cdots+\lambda_{j_{k}}}<\infty
$$

so from Müntz's theorem it follows that $M^{k}(\Lambda)$ is not dense in $C[0,1]$.

Example 6.5. Let $\Lambda:=\left(\lambda_{j}\right)_{j=0}^{\infty}$ be defined by $\lambda_{j}:=j^{2}$. Then

$$
M^{4}(\Lambda) \subset \operatorname{span}\left\{x^{k^{2}+l^{2}+m^{2}+n^{2}}: k, l, m, n \in \mathbb{N}\right\}=\operatorname{span}\left\{x^{n}: n \in \mathbb{N}\right\} .
$$

So in this case the nondenseness of $M^{4}(\Lambda)$ is not obvious at all. 


\section{§7. Multivariate approximation}

In April, 1996, Lorentz sent me a letter related to a volume discussing "multivariate approximation". He speculated: "Some of the chapters may be trivial in the sense that they contain only a collection of known (important) results, others in the sense that their results mimic or are obtainable in a simple way on the univariate material. Even such "trivial" chapters are very much needed. Then there will be chapters (example: multivariate polynomial interpolation) that have very little to do with univariate results. What are these chapters?"

Knowing Lorentz's appreciation of the Remez inequality and its analog for trigonometric polynomials first proved in 51, I believe that Lorentz would like the right higherdimensional analog of Theorem 2.4. This is the only result we formulate in this section; it was proved in 67 .

Let

$$
\mathbf{m}:=\left(m_{1}, m_{2}, \ldots, m_{k}\right) \quad \text { and } \quad \mathbf{j}:=\left(j_{1}, j_{2}, \ldots, j_{k}\right),
$$

where each $m_{j}$ and $j_{\nu}$ is a nonnegative integer. Let

$$
\begin{aligned}
\mathbf{B} & :=\left\{\left(j_{1}, j_{2}, \ldots, j_{k}\right): 1 \leq j_{\nu} \leq m_{\nu}, \nu=1,2, \ldots, k\right\}, \\
\mathbf{x} & :=\left(x_{1}, x_{2}, \ldots, x_{k}\right) \in \mathbb{R}^{k}, \\
\mathbf{d}_{\mathbf{j}} & :=\left(d_{j_{1}}, d_{j_{2}}, \ldots, d_{j_{k}}\right) \in \mathbb{R}^{k}, \quad \mathbf{j} \in \mathbf{B}, \\
G_{\mathbf{m}} & :=\left\{f: f(\mathbf{x})=\sum_{\mathbf{j} \in \mathbf{B}} A_{\mathbf{j}} \exp \left(-\left\|\mathbf{x}-\mathbf{d}_{\mathbf{j}}\right\|\right), A_{\mathbf{j}} \in \mathbb{R}, \quad \mathbf{d}_{\mathbf{j}} \in \mathbb{R}^{k}\right\},
\end{aligned}
$$

where

$$
\left\|\mathbf{x}-\mathbf{d}_{\mathbf{j}}\right\|^{2}:=\sum_{\nu=1}^{k}\left(x_{\nu}-d_{j_{\nu}}\right)^{2}
$$

Theorem 7.1 (Remez-type inequality for $\left.G_{\mathbf{m}}\right)$. Let $s \in(0, \infty)$ and $n \geq 9$. There is an absolute constant $c_{1}>0$ such that

$$
\exp \left(c_{1} R\left(m_{1}, m_{2}, \ldots, m_{k}, s\right)\right) \leq \sup _{f}\|f\|_{\mathbb{R}^{k}} \leq \exp \left(80 R\left(m_{1}, m_{2}, \ldots, m_{k}, s\right)\right),
$$

where

$$
R\left(m_{1}, m_{2}, \ldots, m_{k}, s\right):=\sum_{j=1}^{k}\left(\min \left\{m_{j}^{1 / 2} s^{1 / k}, m_{j} s^{2 / k}\right\}+s^{2 / k}\right),
$$

and the supremum is taken over all $f \in G_{\mathbf{m}}$ with

$$
m\left(\left\{\mathbf{x} \in \mathbb{R}^{k}:|f(\mathbf{x})| \geq 1\right\}\right) \leq s .
$$

\section{§8. Newman's inequality}

Let $\Lambda_{n}:=\left\{\lambda_{0}<\lambda_{1}<\cdots<\lambda_{n}\right\}$ be a set of real numbers. The collection of all linear combinations of $e^{\lambda_{0} t}, e^{\lambda_{1} t}, \ldots, e^{\lambda_{n} t}$ over $\mathbb{R}$ will be denoted by

$$
E\left(\Lambda_{n}\right):=\operatorname{span}\left\{e^{\lambda_{0} t}, e^{\lambda_{1} t}, \ldots, e^{\lambda_{n} t}\right\} .
$$

The elements of $E\left(\Lambda_{n}\right)$ are called exponential sums of $n+1$ terms. For a real-valued function $f$ defined on a set $A$, let

$$
\|f\|_{L_{\infty} A}:=\|f\|_{A}:=\sup \{|f(x)|: x \in A\},
$$

and let

$$
\|f\|_{L_{p} A}:=\left(\int_{A}|f(x)|^{p} d x\right)^{1 / p}, \quad p>0
$$


whenever the Lebesgue integral exists. Newman's inequality (see [114] and [17]) is an essentially sharp Markov-type inequality for $E\left(\Lambda_{n}\right)$ on $[0,1]$ in the case where each $\lambda_{j}$ is nonnegative.

Theorem 8.1 (Newman's inequality). Let $\Lambda_{n}:=\left\{\lambda_{0}<\lambda_{1}<\cdots<\lambda_{n}\right\}$ be a set of nonnegative real numbers. Then

$$
\frac{2}{3} \sum_{j=0}^{n} \lambda_{j} \leq \sup _{0 \neq P \in E\left(\Lambda_{n}\right)} \frac{\left\|P^{\prime}\right\|_{(-\infty, 0]}}{\|P\|_{(-\infty, 0]}} \leq 9 \sum_{j=0}^{n} \lambda_{j} .
$$

Lorentz knew Newman's inequality and he presented the beautiful proof of Newman in the section "Markov-Type Inequalities for Müntz Polynomials" of his book [105, pp. 362-365]. When he learned from me that an extension of P. Borwein's Markov-type inequality [12]

$$
\left\|p^{\prime}\right\|_{[-1,1]} \leq 9 n(k+1)\|p\|_{[-1,1]}
$$

to polynomials from $\mathcal{P}_{n, k}$ (the set of all polynomials of degree at most $n$ with real coefficients and with at most $k(0 \leq k \leq n)$ zeros in the open unit disk) can be obtained easily by using only Lorentz representation and Newman's inequality, he decided to follow my paper [49] to present a proof of (8.1) in his book [105, pp. 64-66], even though my argument gave a multiplicative absolute constant slightly worse than 9. Moreover, Lorentz [105] presented a short and simple proof of the inequality

$$
\left\|p^{(m)}\right\|_{[-1,1]} \leq c(m)(n(k+1))^{m}\|p\|_{[-1,1]}, \quad p \in \mathcal{P}_{n, k},
$$

for higher derivatives, where $c(m)$ is a constant depending only on $m$, as was done (essentially) in [49. Note that a simple induction does not work here due to the lack of a Gauss-Lucas-type theorem.

Lorentz presented Newman's inequality, only with the constant 11 rather than 9 in his book [105]. The book [17] seems to be the first working out the details of the necessary modification and simplification of the proof of Newman's inequality with the constant 9 by eliminating an application of Kolmogorov's inequality from Newman's original approach. Later we observed that the best-known multiplicative constant in Newman's inequality is 8.29 , given in 82 .

In [35], orthonormal Müntz-Legendre polynomials were studied. As a by-product, we proved an essentially sharp version of Newman's inequality in $L_{2}$. An $L_{p}$ version of the upper bound in Newman's inequality was established for $1 \leq p \leq \infty$ in [7, 22] and [55]. Here we formulate some of the main results of 55 that give the constant 8.29 , which is better than the 9 in [7] and [22].

Theorem 8.2. Let $\Lambda_{n}:=\left\{\lambda_{0}<\lambda_{1}<\cdots<\lambda_{n}\right\}$ be a set of nonnegative real numbers. Let $1 \leq p \leq \infty$. Then

$$
\left\|Q^{\prime}\right\|_{L_{p}(-\infty, 0]} \leq 8.29\left(\sum_{j=0}^{n} \lambda_{j}\right)\|Q\|_{L_{p}(-\infty, 0]}
$$

for every $Q \in E\left(\Lambda_{n}\right)$.

Theorem 8.2*. Let $1 \leq p \leq \infty$. Let $\Lambda:=\left(\lambda_{j}\right)_{j=0}^{\infty}$ be a sequence of distinct real numbers greater than $-1 / p$. Then

$$
\left\|x S^{\prime}(x)\right\|_{L_{p}[0,1]} \leq\left(1 / p+8.29\left(\sum_{j=0}^{n}\left(\lambda_{j}+1 / p\right)\right)\right)\|S\|_{L_{p}[0,1]}
$$

for every $S \in M_{n}(\Lambda):=\operatorname{span}\left\{x^{\lambda_{0}}, x^{\lambda_{1}}, \ldots, x^{\lambda_{n}}\right\}$. 
The following $L_{p}[a, b]$ analog of Theorem 8.1, $1 \leq p \leq \infty$, was established in [7].

Theorem 8.3. Let $\Lambda_{n}:=\left\{\lambda_{0}<\lambda_{1}<\cdots<\lambda_{n}\right\}$ be a set of real numbers, and let $1 \leq p \leq \infty, a, b \in \mathbb{R}$, and $a<b$. There is a constant $c_{1}=c_{1}(a, b)$ depending only on $a$ and $b$ such that

$$
\sup _{0 \neq P \in E\left(\Lambda_{n}\right)} \frac{\left\|P^{\prime}\right\|_{L_{p}[a, b]}}{\|P\|_{L_{p}[a, b]}} \leq c_{1}\left(n^{2}+\sum_{j=0}^{n}\left|\lambda_{j}\right|\right) .
$$

Theorem 8.3 had been proved before in 21] $(p=\infty)$ and [55] under the additional assumptions that $\lambda_{j} \geq \delta j$ for each $j$ with a constant $\delta>0$ and with $c_{1}=c_{1}(a, b)$ replaced by $c_{1}=c_{1}(a, b, \delta)$ depending only on $a, b$, and $\delta$. The novelty of Theorem 8.3 was the fact that

$$
\Lambda_{n}:=\left\{\lambda_{0}<\lambda_{1}<\cdots<\lambda_{n}\right\}
$$

is an arbitrary set of real numbers; even the nonnegativity of the exponents $\lambda_{j}$ was not needed.

In 62, the following Nikolski1-Markov-type inequality was proved for $E\left(\Lambda_{n}\right)$ in the case of $(-\infty, 0]$.

Theorem 8.4. Let $\Lambda_{n}:=\left\{\lambda_{0}<\lambda_{1}<\cdots<\lambda_{n}\right\}$ be a set of nonnegative real numbers, and let $0<q \leq p \leq \infty$. Let $\mu$ be a nonnegative integer. There are constants $c_{2}=$ $c_{2}(p, q, \mu)>0$ and $c_{3}=c_{3}(p, q, \mu)$ depending only on $p, q$, and $\mu$ such that

$$
c_{2}\left(\sum_{j=0}^{n} \lambda_{j}\right)^{\mu+\frac{1}{q}-\frac{1}{p}} \leq \sup _{0 \neq P \in E\left(\Lambda_{n}\right)} \frac{\left\|P^{(\mu)}\right\|_{L_{p}(-\infty, 0]}}{\|P\|_{L_{q}(-\infty, 0]}} \leq c_{3}\left(\sum_{j=0}^{n} \lambda_{j}\right)^{\mu+\frac{1}{q}-\frac{1}{p}},
$$

where the lower bound holds for all $0<q \leq p \leq \infty$ and $\mu \geq 0$, while the upper bound holds when $\mu=0$ and $0<q \leq p \leq \infty$, and when $\mu \geq 1, p \geq 1$, and $0<q \leq p \leq \infty$. Also, there are constants $c_{2}=c_{2}(q, \mu)>0$ and $c_{3}=c_{3}(q, \mu)$ depending only on $q$ and $\mu$ such that

$$
c_{2}\left(\sum_{j=0}^{n} \lambda_{j}\right)^{\mu+\frac{1}{q}} \leq \sup _{0 \neq P \in E\left(\Lambda_{n}\right)} \frac{\left|P^{(\mu)}(y)\right|}{\|P\|_{L_{q}(-\infty, y]}} \leq c_{3}\left(\sum_{j=0}^{n} \lambda_{j}\right)^{\mu+\frac{1}{q}}
$$

for all $0<q \leq \infty, \mu \geq 1$, and $y \in \mathbb{R}$.

Motivated by a question of Michel Weber, in [66] we proved the following couple of theorems.

Theorem 8.5. Suppose $0<q \leq p \leq \infty, a, b \in \mathbb{R}$, and $a<b$. There are constants $c_{4}=c_{4}(p, q, a, b)>0$ and $c_{5}=c_{5}(p, q, a, b)$ depending only on $p, q, a$, and $b$ such that

$$
c_{4}\left(n^{2}+\sum_{j=0}^{n}\left|\lambda_{j}\right|\right)^{\frac{1}{q}-\frac{1}{p}} \leq \sup _{0 \neq P \in E\left(\Lambda_{n}\right)} \frac{\|P\|_{L_{p}[a, b]}}{\|P\|_{L_{q}[a, b]}} \leq c_{5}\left(n^{2}+\sum_{j=0}^{n}\left|\lambda_{j}\right|\right)^{\frac{1}{q}-\frac{1}{p}} .
$$

Theorem 8.6. Suppose $0<q \leq p \leq \infty, a, b \in \mathbb{R}$, and $a<b$. There are constants $c_{6}=c_{6}(p, q, a, b)>0$ and $c_{7}=c_{7}(p, q, a, b)$ depending only on $p, q, a$, and $b$ such that

$$
c_{6}\left(n^{2}+\sum_{j=0}^{n}\left|\lambda_{j}\right|\right)^{1+\frac{1}{q}-\frac{1}{p}} \leq \sup _{0 \neq P \in E\left(\Lambda_{n}\right)} \frac{\left\|P^{\prime}\right\|_{L_{p}[a, b]}}{\|P\|_{L_{q}[a, b]}} \leq c_{7}\left(n^{2}+\sum_{j=0}^{n}\left|\lambda_{j}\right|\right)^{1+\frac{1}{q}-\frac{1}{p}},
$$

where the lower bound holds for all $0<q \leq p \leq \infty$, while the upper bound holds when $p \geq 1$ and $0<q \leq p \leq \infty$. 
It was observed by Bernstein that Markov's inequality for monotone polynomials is not essentially better than that for all polynomials. He proved that

$$
\sup _{p} \frac{\left\|p^{\prime}\right\|_{[-1,1]}}{\|p\|_{[-1,1]}}= \begin{cases}\frac{1}{4}(n+1)^{2} & \text { if } n \text { is odd } \\ \frac{1}{4} n(n+2) & \text { if } n \text { is even, }\end{cases}
$$

where the supremum is taken for all polynomials $0 \neq p$ of degree at most $n$ that are monotone on $[-1,1]$. See [121, p. 607], for instance.

In 68, an effort was made to extend the above results of Bernstein to the classes $E\left(\Lambda_{n}\right)$. We proved the following couple of results.

Theorem 8.7. Let $n \geq 2$ be an integer, and let $b \in \mathbb{R}$. Then there is an absolute constant $c_{1}>0$ such that

$$
\frac{c_{1}}{\log n} \sum_{j=0}^{n} \lambda_{j} \leq \sup _{P} \frac{\left\|P^{\prime}\right\|_{(-\infty, b]}}{\|P\|_{(-\infty, b]}} \leq 9 \sum_{j=0}^{n} \lambda_{j},
$$

where the supremum is taken for all $0 \neq P \in E\left(\Lambda_{n}\right)$ increasing on $(-\infty, \infty)$.

Theorem 8.8. Let $n \geq 2$ be an integer. Let $[a, b]$ be a finite interval with length $b-a>0$. There are positive constants $c_{2}=c_{2}(a, b)$ and $c_{3}=c_{3}(a, b)$ depending only on $a$ and $b$ such that

$$
c_{2}\left(n^{2}+\frac{1}{\log n} \sum_{j=0}^{n}\left|\lambda_{j}\right|\right) \leq \sup _{P} \frac{\left\|P^{\prime}\right\|_{[a, b]}}{\|P\|_{[a, b]}} \leq c_{3}\left(n^{2}+\sum_{j=0}^{n}\left|\lambda_{j}\right|\right),
$$

where the supremum is taken for all $0 \neq P \in E\left(\Lambda_{n}\right)$ increasing on $(-\infty, \infty)$.

It is expected that the factor $1 / \log n$ in the above theorems can be dropped.

Most of the results in this section are fairly recent. I think Lorentz would like the results in this section and might include some of them rather than Theorems 8.3 and 8.4 in [105, p. 367] with proofs.

\section{§9. LitTLEWOOD POLYNOMIALS}

The well-known Littlewood conjecture was solved by Konyagin [93] and independently by McGehee, Pigno, and B. Smith 107. Based on these, Lorentz worked out a textbook proof of the conjecture in [44.

Theorem 9.1. Let $n_{1}, n_{2}, \ldots, n_{N}$ be distinct integers. For some absolute constant $c>0$,

$$
\int_{0}^{2 \pi}\left|\sum_{k=1}^{N} e^{i n_{k} t}\right| d t \geq c \log N
$$

This is an obvious consequence of the following.

Theorem 9.2. Let $n_{1}<n_{2}<\cdots<n_{N}$ be integers. Let $a_{1}, a_{2}, \ldots, a_{k}$ be arbitrary complex numbers. We have

$$
\int_{0}^{2 \pi}\left|\sum_{k=1}^{N} a_{k} e^{i n_{k} t}\right| d t \geq \frac{1}{30} \sum_{k=1}^{N} \frac{\left|a_{k}\right|}{k} .
$$

I read the proof of Theorem 9.2 presented in Lorentz's book [4] with special interest. This was one of my main motivations to start working on unimodular polynomials (polynomials with complex coefficients of modulus 1), and Littlewood polynomials (polynomials with coefficients from $\{-1,1\}$ ), and with other classes of polynomials with various other coefficient constraints.

Pichorides, who contributed essentially to the proof of the Littlewood conjecture, observed in [119] that the original Littlewood conjecture (when all the coefficients are 
from $\{0,1\})$ would follow from a result on the $L_{1}$-norm of such polynomials on sets $E \subset \partial D$ of measure $\pi$. Namely, if

$$
\int_{E}\left|\sum_{j=0}^{n} z^{k_{j}}\right||d z| \geq c
$$

for any subset $E \subset \partial D$ of measure $\pi$ with an absolute constant $c>0$, then the original Littlewood conjecture holds. Throughout this section the measure of a set $E \subset \partial D$ is the linear Lebesgue measure of the set

$$
\left\{t \in[-\pi, \pi): e^{i t} \in E\right\} .
$$

Konyagin 92 gave a lovely probabilistic proof showing that this hypothesis fails. He did however conjecture the following: for any fixed set $E \subset \partial D$ of positive measure there exists a constant $c=c(E)>0$ depending only on $E$ such that

$$
\int_{E}\left|\sum_{j=0}^{n} z^{k_{j}}\right||d z| \geq c(E)
$$

In other words, the sets $E_{\varepsilon} \subset \partial D$ of measure $\pi$ in his example where

$$
\int_{E_{\varepsilon}}\left|\sum_{j=0}^{n} z^{k_{j}}\right||d z|<\varepsilon
$$

must vary with $\varepsilon>0$.

In [29] we showed, among other things, that Konyagin's conjecture holds on subarcs of the unit circle $\partial D$.

In 84, C. S. Güntürk constructed certain types of near-optimal approximations of a class of functions analytic in the unit disk by power series with two distinct coefficients. More precisely, it was shown that if all the coefficients of the power series $f(z)$ are real and lie in $[-\mu, \mu]$, where $\mu<1$, then there exists a power series $Q(z)$ with coefficients in $\{-1,+1\}$ such that $|f(z)-Q(z)| \rightarrow 0$ at the rate $\exp \left(C|1-z|^{-1}\right)$ as $z \rightarrow 1$ nontangentially inside the unit disk. Güntürk refers to P. Borwein, Erdélyi, and Kós in [34 to see that this type of decay rate is best possible. The special case of $f \equiv 0$ yields a near-optimal solution to the "fair duel problem" of Konyagin, as it was described in the Introduction of 84 .

In [34, we considered the problem of minimizing the uniform norm on $[0,1]$ over polynomials $0 \neq p$ of the form

$$
p(x)=\sum_{j=0}^{n} a_{j} x^{j}, \quad\left|a_{j}\right| \leq 1, \quad a_{j} \in \mathbb{C},
$$

where the modulus of the first nonzero coefficient is at least $\delta>0$. Essentially sharp bounds were given for this problem. An interesting related result states that there are absolute constants $c_{1}>0$ and $c_{2}>0$ such that

$$
\exp \left(-c_{1} \sqrt{n}\right) \leq \inf _{0 \neq p \in \mathcal{F}_{n}}\|p\|_{[0,1]} \leq \exp \left(-c_{2} \sqrt{n}\right)
$$

for every $n \geq 2$, where $\mathcal{F}_{n}$ denotes the set of polynomials of degree at most $n$ and with coefficients in $\{-1,0,1\}$.

The results of [29] show that many types of polynomials cannot be small on subarcs of the unit circle in the complex plane. A typical result of [29] is the following. There are absolute constants $c_{1}>0, c_{2}>0$, and $c_{3}>0$ such that

$$
\exp \left(-c_{1} / a\right) \leq \inf _{0 \neq p \in \mathcal{F}_{n}}\|p\|_{L_{1}(A)}, \inf _{0 \neq p \in \mathcal{F}_{n}}\|p\|_{A} \leq \exp \left(-c_{2} / a\right)
$$

for every subarc $A$ of the unit circle $\partial D:=\{z \in \mathbb{C}:|z|=1\}$ with length $0<a<c_{3}$. 
The lower bound results extend to the class of $f$ of the form

$$
f(z)=\sum_{j=m}^{n} a_{j} z^{j}, \quad a_{j} \in \mathbb{C}, \quad\left|a_{j}\right| \leq M, \quad\left|a_{m}\right|=1,
$$

with varying nonnegative integers $m \leq n$. In [29, it was also shown that functions $f$ of the above form cannot be arbitrarily small uniformly on subarcs of the circle. However, this does not extend to sets of positive measure. It was shown that it is possible to find a polynomial of the above form that is arbitrarily small on as much of the boundary (in the sense of linear Lebesgue measure) as one likes.

The height of a polynomial

$$
p_{n}(z):=\sum_{j=0}^{n} a_{j} z^{j}, \quad a_{j} \in \mathbb{C}, \quad a_{n} \neq 0,
$$

is defined by

$$
H\left(p_{n}\right):=\max \left\{\frac{\left|a_{j}\right|}{\left|a_{n}\right|}: j=0,1, \ldots, n\right\} .
$$

An easy way to formulate a corollary to the results of [29] is the following.

Corollary 9.3. Let $A$ be a subarc of the unit circle with length $\ell(A)=a$. If $\left(p_{k}\right)$ is a sequence of monic polynomials that tends to 0 in $L_{1}(A)$, then the sequence $H\left(p_{k}\right)$ of heights tends to $\infty$.

In 24], we were concerned with the problem of minimizing the supremum norm, on an interval, of a nonzero polynomial of degree at most $n$ with integral coefficients. This is an old and hard problem that cannot be solved exactly in any nontrivial cases. See the references in [24]. We examined the case of the interval $[0,1]$ in most detail. We improved the known bounds by a small but interesting amount. This allowed us to garner further information about the structure of such minimal polynomials and their factors. This was primarily a (substantial) computational exercise. We also examined some of the structure of such minimal "integer Chebyshev" polynomials. We disproved conjecture 36 (ascribed to the Chudnovsky brothers and others) in [111, p. 201]. In recent years a number of papers related to 24 were published. See [39], 86], and [138, for example, and the references therein.

In 1945, Duffin and Schaeffer proved that any power series that is bounded in a sector of the open unit disk and has coefficients from a finite subset of $\mathbb{C}$ is already a rational function. Their proof is relatively indirect. It is one purpose of [37] to give a shorter direct proof of this beautiful and surprising theorem. An easy consequence of this, for example, is that any algebraic function that has a power series expansion on the open unit disk with coefficients from a finite subset of $\mathbb{C}$ is, in fact, a rational function.

\section{§10. INEQUALITIES FOR GENERALIZED POLYNOMIALS}

The function

$$
f(x):=|\omega| \prod_{j=1}^{m}\left|x-z_{j}\right|^{r_{j}}
$$

with $0<r_{j} \in \mathbb{R}, z_{j} \in \mathbb{C}$, and $0 \neq \omega \in \mathbb{C}$ is called a generalized (algebraic) polynomial of degree $N:=\sum_{j=1}^{m} r_{j}$. If $f$ is a positive constant identically, its degree is defined to be 0 , while if $f$ is identically 0 , its degree is defined to be -1 . Let $\mathrm{GAP}_{N}$ be the set of all generalized algebraic polynomials of degree at most $N$. If in the representation (10.1) of $f$ all the exponents $r_{j}$ are integers, then $f$ is the absolute value of a usual algebraic polynomial (of degree $N$ ). If $f \in \mathrm{GAP}_{N}$ is of the form (10.1) with distinct $z_{j} \in \mathbb{C}$, then 
the numbers $z_{j}$ are called the zeros of $f$, while the exponent $r_{j}$ is called the multiplicity of the zero $z_{j}$ in $f$.

The function

$$
P(x):=a_{0}+\sum_{k=1}^{n}\left(a_{k} \cos k x+b_{k} \sin k x\right), \quad a_{k}, b_{k} \in \mathbb{R}, \quad a_{n} b_{n} \neq 0,
$$

is called a real trigonometric polynomial of degree $n$. It is well known that every real trigonometric polynomial $P$ of degree (order) $n$ can be written as

$$
P(x)=\omega \prod_{j=1}^{2 n} \sin \left(\left(x-z_{j}\right) / 2\right),
$$

where $\omega \in \mathbb{R}, z_{j} \in \mathbb{C}$, and the nonreal zeros $z_{j}$ of $P$ form conjugate pairs. The function

$$
P(x):=\omega \prod_{j=1}^{m}\left|\sin \left(\left(x-z_{j}\right) / 2\right)\right|^{r_{j}}, \quad x \in \mathbb{R},
$$

where $0<r_{j} \in \mathbb{R}, z_{j} \in \mathbb{C}$ are distinct $(\bmod 2 \pi)$, and $0<\omega \in \mathbb{R}$, is called a generalized trigonometric polynomial of degree $N:=\frac{1}{2} \sum_{j=1}^{m} r_{j}$. If $P$ is a constant identically, then its degree is defined to be 0 . Note that the absolute value of a real trigonometric polynomial of degree $n$ may be viewed as a generalized trigonometric polynomial of degree $n$. Let $\mathrm{GTP}_{N}$ denote the set of all generalized trigonometric polynomials of degree at most $N$. Observe that if $P \in \mathrm{GTP}_{N}$ is of the form (10.2), then

$$
P(x):=\omega \prod_{j=1}^{s}\left(\sin \left(\left(x-z_{j}\right) / 2\right) \sin \left(\left(x-\bar{z}_{j}\right) / 2\right)\right)^{r_{j} / 2}=\prod_{j=1}^{s} T_{j}(x)^{r_{j} / 2}, \quad x \in \mathbb{R},
$$

where each $T_{j}$ is a real trigonometric polynomial of degree 1 that is nonnegative on the real line. For $P \in \mathrm{GTP}_{N}$ of the form (10.2), the numbers $z_{j}$ are called the zeros of $P$, while the exponent $r_{j}$ is called the multiplicity of the zero $z_{j}$ in $P$.

The problem arises how to define $f^{\prime}$ for an $f \in \operatorname{GAP}_{N}$ and $P^{\prime}$ for a $P \in \mathrm{GTP}_{N}$. Observe that if $r_{j} \geq 1$ for each $j=1,2, \ldots, m$ in (10.2), then, although $P^{\prime}$ may not exist at the zeros of $P$, the one-sided derivatives $P_{-}^{\prime}$ and $P_{+}^{\prime}$ exist, and their absolute values are equal. This means that $\left|P^{\prime}\right|$ is well defined on the real line by either $\left|P_{-}^{\prime}\right|$ or $\left|P_{+}^{\prime}\right|$. Similarly, if $r_{j} \geq 1$ for each $j=1,2, \ldots, m$ in (10.1), then $\left|f^{\prime}\right|$ is well defined on the real line. It is a simple exercise to check that if $P \in \mathrm{GTP}_{N}$ has only real zeros with multiplicities at least 1 , then $\left|P^{\prime}\right| \in \mathrm{GTP}_{N}$ has only real zeros as well, and at least one of any two adjacent zeros of $\left|P^{\prime}\right|$ has multiplicity exactly 1 . A similar comment can be made on $f \in \operatorname{GAP}_{N}$ having only real zeros with multiplicities at least 1.

One might expect to extend various polynomial inequalities to generalized polynomials by writing the generalized degree $N$ in place of the usual degree $n$. However, even when it is possible, such an extension might be far from obvious. Remez-type inequalities serve as examples of a situation where a polynomial inequality easily extends to generalized polynomials.

In [76, we started with the (straightforward) extension of the algebraic and trigonometric Remez-type inequalities to $\mathrm{GAP}_{N}$ and $\mathrm{GTP}_{N}$ and proved the following results.

Theorem 10.1. There is an absolute constant $0<c_{1}<1$ such that

$$
m\left(y \in[-1,1]: f(y) \geq \exp (-N \sqrt{s}) \max _{-1 \leq x \leq 1} f(x)\right) \geq c_{1} s
$$

for every $f \in \mathrm{GAP}_{N}$ and $0<s<2$. 
Theorem 10.2. There is an absolute constant $0<c_{2}<1$ such that

$$
m\left(t \in[-\pi, \pi]: P(t) \geq \exp (-N s) \max _{-\pi \leq \tau \leq \pi} P(\tau)\right) \geq c_{2} s
$$

for every $P \in \operatorname{GTP}_{N}$ and $0<s<2 \pi$.

As a consequence, in [76, we obtained the Nikolskil-type inequalities below.

Theorem 10.3. Let $\chi$ be a nonnegative, nondecreasing function defined on $[0, \infty)$ such that $\chi(x) / x$ is nonincreasing in $[0, \infty)$. Then for $0<q<p \leq \infty$ we have

$$
\|\chi(P)\|_{L_{p}[-\pi, \pi]} \leq\left(c_{3}(1+q N)\right)^{1 / q-1 / p}\|\chi(P)\|_{L_{q}[-\pi, \pi]}, \quad P \in \operatorname{GTP}_{N},
$$

where $c_{3}$ is an absolute constant. If $\chi(x)=x$, then $c_{3}=e(4 \pi)^{-1}$ is suitable.

Theorem 10.4. Let $\chi$ be a nonnegative, nondecreasing function defined on $[0, \infty)$ such that $\chi(x) / x$ is nonincreasing in $[0, \infty)$. Then for $0<q<p \leq \infty$ we have

$$
\|\chi(f)\|_{L_{p}[-1,1]} \leq\left(c_{4}(1+q N)\right)^{2 / q-2 / p}\|\chi(f)\|_{L_{q}[-1,1]}, \quad f \in \mathrm{GAP}_{N},
$$

where $c_{4}$ is an absolute constant. If $\chi(x)=x$, then $c_{4}=e^{2}(2 \pi)^{-1}$ is suitable.

In [50] and [76, we proved even (essentially) sharp Markov- and Bernstein-type inequalities for the classes $\mathrm{GAP}_{N}$ and $\mathrm{GTP}_{N}$ in $L_{p}$ norms. In [77, we extended these results to the setting below.

Theorem 10.5. Let $0<p \leq \infty$. We have

$$
\left\|P^{\prime} Q\right\|_{L_{p}[-\pi, \pi]} \leq c^{1+1 / p}(N+M) \log (\min (N, M+1)+1)\|P Q\|_{L_{p}[-\pi, \pi]}
$$

for any two $P \in \mathrm{GTP}_{N}$ and $Q \in \mathrm{GTP}_{M}$ such that the roots of $P$ and $Q$ have multiplicities at least 1 . Moreover, this inequality is sharp up to the constant $c^{1+1 / p}$.

Theorem 10.6. Let $0<p \leq \infty$. We have

$$
\left\|\sqrt{1-x^{2}} f^{\prime}(x) g(x)\right\|_{L_{p}[-1,1]} \leq c^{1+1 / p}(N+M) \log (\min (N, M+1)+1)\|f g\|_{L_{p}[-1,1]}
$$

for any two $f \in \mathrm{GAP}_{N}$ and $g \in \mathrm{GAP}_{M}$ such that the roots of $f$ and $g$ have multiplicities at least 1 . This inequality is sharp up to the constant $c^{1+1 / p}$.

Theorem 10.7. Let $0<p \leq \infty$. We have

$$
\left\|f^{\prime} g\right\|_{L_{p}[-1,1]} \leq c^{1+1 / p}(N+M)^{2}\|f g\|_{L_{p}[-1,1]}
$$

for any two $f \in \operatorname{GAP}_{N}$ and $g \in \mathrm{GAP}_{M}$ such that the roots of $f$ have multiplicities at least 1 . This inequality is sharp up to the factor $c^{1+1 / p}$ for all $N, M \geq 1$.

In [74, we combined some of our inequalities for generalized polynomials with some other ideas and obtained the following result.

Theorem 10.8. For all Jacobi weight functions $w(x)=(1-x)^{\alpha}(1+x)^{\beta}$ with $\alpha \geq-1 / 2$ and $\beta \geq-1 / 2$, the inequalities

$$
\max _{x \in[-1,1]} \frac{p_{n}^{2}(w, x)}{\sum_{k=0}^{n} p_{k}^{2}(w, x)} \leq \frac{4\left(2+\sqrt{\alpha^{2}+\beta^{2}}\right)}{2 n+\alpha+\beta+2}
$$

and

$$
\max _{x \in[-1,1]} \sqrt{1-x^{2}} w(x) p_{n}^{2}(w, x) \leq \frac{2 e\left(2+\sqrt{\alpha^{2}+\beta^{2}}\right)}{\pi}
$$

hold for $n=0,1, \ldots$, where the $p_{n}(w, x)$ denote the orthonormal polynomials of degree $n$ associated with the weight $w$ on $[-1,1]$. 


\section{§11. MARKOV- AND BERnstein-TyPe InEQUALITIES FOR RATIONAL FUnCTIONS}

We denote by $\mathcal{P}_{n}^{r}$ and $\mathcal{P}_{n}^{c}$ the sets of all algebraic polynomials of degree at most $n$ with real or complex coefficients, respectively. The sets of all trigonometric polynomials of degree at most $n$ with real or complex coefficients, respectively, are denoted by $\mathcal{T}_{n}^{r}$ and $\mathcal{T}_{n}^{c}$. We will use the notation

$$
\|f\|_{A}=\sup _{z \in A}|f(z)|
$$

for continuous functions $f$ defined on $A$. Let

$$
D:=\{z \in \mathbb{C}:|z| \leq 1\}, \quad \partial D:=\{z \in \mathbb{C}:|z|=1\}, \quad K:=\mathbb{R} \quad(\bmod 2 \pi) .
$$

The classical inequalities of Bernstein [8] state that

$$
\begin{array}{rlrl}
\left|p^{\prime}\left(z_{0}\right)\right| & \leq n\|p\|_{\partial D}, & p \in \mathcal{P}_{n}^{c}, & z_{0} \in \partial D, \\
\left|t^{\prime}\left(\theta_{0}\right)\right| \leq n\|t\|_{K}, & & t \in \mathcal{T}_{n}^{c}, & \theta_{0} \in K, \\
\left|p^{\prime}\left(x_{0}\right)\right| \leq \frac{n}{\sqrt{1-x_{0}^{2}}}\|p\|_{[-1,1]}, & p \in \mathcal{P}_{n}^{c}, & x_{0} \in(-1,1) .
\end{array}
$$

Proofs of the above inequalities can be found in almost every book on approximation theory; see [104, for instance. An extensive study of Markov- and Bernstein-type inequalities is presented in [120, [121, and [17].

In [19], we study the following rational function spaces:

$$
\mathcal{P}_{n}^{c}\left(a_{1}, a_{2}, \ldots, a_{n} ; \partial D\right):=\left\{\frac{p_{n}(z)}{\prod_{j=1}^{n}\left(z-a_{j}\right)}: p_{n} \in \mathcal{P}_{n}^{c}\right\}
$$

on $\partial D$ with $\left\{a_{1}, a_{2}, \ldots, a_{n}\right\} \subset \mathbb{C} \backslash \partial D$;

$$
\mathcal{T}_{n}^{c}\left(a_{1}, a_{2}, \ldots, a_{2 n} ; K\right):=\left\{\frac{t_{n}(\theta)}{\prod_{j=1}^{2 n} \sin \left(\left(\theta-a_{j}\right) / 2\right)}: t_{n} \in \mathcal{T}_{n}^{c}\right\}
$$

on $K$ with $\left\{a_{1}, a_{2}, \ldots, a_{2 n}\right\} \subset \mathbb{C} \backslash \mathbb{R}$;

$$
\mathcal{P}_{n}^{c}\left(a_{1}, a_{2}, \ldots, a_{n} ;[-1,1]\right):=\left\{\frac{p_{n}(x)}{\prod_{j=1}^{n}\left(x-a_{j}\right)}: p_{n} \in \mathcal{P}_{n}^{c}\right\}
$$

on $[-1,1]$ with $\left\{a_{1}, a_{2}, \ldots, a_{n}\right\} \subset \mathbb{C} \backslash[-1,1]$;

$$
\mathcal{P}_{n}^{c}\left(a_{1}, a_{2}, \ldots, a_{n} ; \mathbb{R}\right):=\left\{\frac{p_{n}(x)}{\prod_{j=1}^{n}\left(x-a_{j}\right)}: p_{n} \in \mathcal{P}_{n}^{c}\right\}
$$

on $\mathbb{R}$ with $\left\{a_{1}, a_{2}, \ldots, a_{n}\right\} \subset \mathbb{C} \backslash \mathbb{R}$; and

$$
\mathcal{P}_{n}^{r}\left(a_{1}, a_{2}, \ldots, a_{n} ; \mathbb{R}\right):=\left\{\frac{p_{n}(x)}{\prod_{j=1}^{n}\left|x-a_{j}\right|}: p_{n} \in \mathcal{P}_{n}^{r}\right\}
$$

on $\mathbb{R}$ with $\left\{a_{1}, a_{2}, \ldots, a_{n}\right\} \subset \mathbb{C} \backslash \mathbb{R}$.

The spaces

$$
\mathcal{T}_{n}^{r}\left(a_{1}, a_{2}, \ldots, a_{2 n} ; K\right):=\left\{\frac{t_{n}(\theta)}{\prod_{j=1}^{2 n}\left|\sin \left(\left(\theta-a_{j}\right) / 2\right)\right|}: t_{n} \in \mathcal{T}_{n}^{r}\right\}
$$

on $K$ with $\left\{a_{1}, a_{2}, \ldots, a_{n}\right\} \subset \mathbb{C} \backslash \mathbb{R}$ and

$$
\mathcal{P}_{n}^{r}\left(a_{1}, a_{2}, \ldots, a_{n} ;[-1,1]\right):=\left\{\frac{p_{n}(x)}{\prod_{j=1}^{n}\left|x-a_{j}\right|}: p_{n} \in \mathcal{P}_{n}^{r}\right\}
$$


on $[-1,1]$ with $\left\{a_{1}, a_{2}, \ldots, a_{n}\right\} \subset \mathbb{C} \backslash[-1,1]$ were studied in [36] and [17, and the sharp Bernstein-Szegö type inequalities

$$
f^{\prime}\left(\theta_{0}\right)^{2}+\widetilde{B}_{n}\left(\theta_{0}\right)^{2} f\left(\theta_{0}\right)^{2} \leq \widetilde{B}\left(\theta_{0}\right)^{2}\|f\|_{K}^{2}, \quad \theta_{0} \in K,
$$

for every $f \in \mathcal{T}_{n}^{r}\left(a_{1}, a_{2}, \ldots, a_{2 n} ; K\right)$ with

$$
\left(a_{1}, a_{2}, \ldots, a_{2 n}\right) \subset \mathbb{C} \backslash \mathbb{R}, \quad \operatorname{Im}\left(a_{j}\right)>0, \quad j=1,2, \ldots, 2 n,
$$

and

$$
\left(1-x_{0}^{2}\right) f^{\prime}\left(x_{0}\right)^{2}+B_{n}\left(x_{0}\right)^{2} f\left(x_{0}\right)^{2} \leq B_{n}\left(x_{0}\right)^{2}\|f\|_{[-1,1]}^{2}, \quad x_{0} \in(-1,1),
$$

for every $f \in \mathcal{P}_{n}^{r}\left(a_{1}, a_{2}, \ldots, a_{n} ;[-1,1]\right)$ with

$$
\left\{a_{1}, a_{2}, \ldots, a_{n}\right\} \subset \mathbb{C} \backslash[-1,1]
$$

were proved, where

$$
\widetilde{B}_{n}(\theta):=\frac{1}{2} \sum_{j=1}^{2 n} \frac{1-\left|e^{i a_{j}}\right|^{2}}{\left|e^{i a_{j}}-e^{i \theta}\right|^{2}}, \quad \theta \in K,
$$

and

$$
B_{n}(x):=\operatorname{Re}\left(\sum_{j=1}^{n} \frac{\sqrt{a_{j}^{2}-1}}{a_{j}-x}\right), \quad x \in[-1,1],
$$

with the choice of $\sqrt{a_{j}^{2}-1}$ determined by

$$
\left|a_{j}-\sqrt{a_{j}^{2}-1}\right|<1 .
$$

These inequalities give sharp upper bounds for $\left|f^{\prime}\left(\theta_{0}\right)\right|$ and $\left|f^{\prime}\left(x_{0}\right)\right|$ only at $n$ points in $K$ and $[-1,1]$, respectively. In [19], we established Bernstein-type inequalities for the spaces

$$
\mathcal{P}_{n}^{c}\left(a_{1}, a_{2}, \ldots, a_{n}, \partial D\right) \text { and } \mathcal{T}_{n}^{c}\left(a_{1}, a_{2}, \ldots, a_{2 n} ; K\right),
$$

which are sharp at every $z \in \partial D$ and $\theta \in K$, respectively. An essentially sharp Bernsteintype inequality was also established for the space

$$
\mathcal{P}_{n}^{c}\left(a_{1}, a_{2}, \ldots, a_{;}[-1,1]\right) .
$$

A Bernstein-type inequality of Russak [118 was extended to the spaces

$$
\mathcal{P}_{n}^{c}\left(a_{1}, a_{2}, \ldots, a_{n} ; \mathbb{R}\right),
$$

and a Bernstein-Szegö type inequality was established for the spaces

$$
\mathcal{P}_{n}^{r}\left(a_{1}, a_{2}, \ldots, a_{n} ; \mathbb{R}\right) .
$$

For a polynomial

$$
q_{n}(z)=c \prod_{j=1}^{n}\left(z-a_{j}\right), \quad 0 \neq c \in \mathbb{C}, \quad a_{j} \in \mathbb{C},
$$

we define

$$
q_{n}^{*}(z)=\bar{c} \prod_{j=1}^{n}\left(1-\bar{a}_{j} z\right)=z^{n} \bar{q}_{n}\left(z^{-1}\right) .
$$

It is well known and easy to check that

$$
\left|q_{n}(z)\right|=\left|q_{n}^{*}(z)\right|, \quad z \in \partial D .
$$


We also define the Blaschke products

$$
S_{n}(z):=\prod_{j=1}^{n} \frac{1-\bar{a}_{j} z}{z-a_{j}}
$$

associated with $\left\{a_{1}, a_{2}, \ldots, a_{n}\right\} \subset \mathbb{C} \backslash \partial D$, and

$$
\widetilde{S}_{n}(z):=\prod_{j=1}^{n} \frac{z-\bar{a}_{j}}{z-a_{j}}
$$

associated with $\left\{a_{1}, a_{2}, \ldots, a_{n}\right\} \subset \mathbb{C} \backslash \mathbb{R}$.

In [19], we proved the following five theorems. The first of them was called the "Borwein-Erdélyi inequality" in [130.

Theorem 11.1. Let $\left\{a_{1}, a_{2}, \ldots, a_{n}\right\} \subset \mathbb{C} \backslash \partial D$. Then

$$
\left|f^{\prime}\left(z_{0}\right)\right| \leq \max \left\{\sum_{\substack{j=1 \\\left|a_{j}\right|>1}} \frac{\left|a_{j}\right|^{2}-1}{\left|a_{j}-z_{0}\right|^{2}}, \sum_{\substack{j=1 \\\left|a_{j}\right|<1}} \frac{1-\left|a_{j}\right|^{2}}{\left|a_{j}-z_{0}\right|^{2}}\right\}\|f\|_{\partial D}
$$

for every $f \in \mathcal{P}_{n}^{c}\left(a_{1}, a_{2}, \ldots, a_{n} ; \partial D\right)$ and $z_{0} \in \partial D$. If the first sum is not less than the second sum for a fixed $z_{0} \in \partial D$, then equality occurs for $f=c S_{n}^{+}, c \in \mathbb{C}$, where $S_{n}^{+}$ is the Blaschke product associated with those $a_{j}$ for which $\left|a_{j}\right|>1$. If the first sum is not greater than the second sum for a fixed $z_{0} \in \partial D$, then equality occurs for $f=c S_{n}^{-}$, $c \in \mathbb{C}$, where $S_{n}^{-}$is the Blaschke product associated with those $a_{j}$ for which $\left|a_{j}\right|<1$.

Theorem 11.2. Let $\left\{a_{1}, a_{2}, \ldots, a_{2 n}\right\} \subset \mathbb{C} \backslash \mathbb{R}$. Then

$$
\left|f^{\prime}\left(\theta_{0}\right)\right| \leq \max \left\{\sum_{\substack{j=1 \\ \operatorname{Im}\left(a_{j}\right)<0}}^{2 n} \frac{\left|e^{i a_{j}}\right|^{2}-1}{\left|e^{i a_{j}}-e^{i \theta_{0}}\right|^{2}}, \sum_{\substack{j=1 \\ \operatorname{Im}\left(a_{j}\right)>0}}^{2 n} \frac{1-\left|e^{i a_{j}}\right|^{2}}{\left|e^{i a_{j}}-e^{i \theta_{0}}\right|^{2}}\right\}\|f\|_{K}
$$

for every $f \in \mathcal{T}_{n}^{c}\left(a_{1}, a_{2}, \ldots, a_{2 n} ; K\right)$ and $\theta_{0} \in K$. If the first sum is not less than the second sum for a fixed $\theta_{0} \in K$, then equality occurs for $f(\theta)=c S_{2 n}^{+}\left(e^{i \theta}\right), c \in \mathbb{C}$. If the first sum is not greater than the second sum for a fixed $\theta_{0} \in K$, then equality occurs for $f(\theta)=c S_{2 n}^{-}\left(e^{i \theta}\right), \quad c \in \mathbb{C}$. The products $S_{2 n}^{+}$and $S_{2 n}^{-}$associated with $\left\{e^{i a_{1}}, e^{i a_{2}}, \ldots, e^{i a_{2 n}}\right\}$ are defined as in Theorem 11.1.

Theorem 11.3. Let $\left\{a_{1}, a_{2}, \ldots, a_{n}\right\} \subset \mathbb{C} \backslash[-1,1]$, and let

$$
c_{j}:=a_{j}-\sqrt{a_{j}^{2}-1}, \quad\left|c_{j}\right|<1,
$$

with the choice of root in $\sqrt{a_{j}^{2}-1}$ determined by $\left|c_{j}\right|<1$. Then

$$
\left|f^{\prime}\left(x_{0}\right)\right| \leq \frac{1}{\sqrt{1-x_{0}^{2}}} \max \left\{\sum_{j=1}^{n} \frac{\left|c_{j}\right|^{-2}-1}{\left|c_{j}^{-1}-z_{0}\right|^{2}}, \sum_{j=1}^{n} \frac{1-\left|c_{j}\right|^{2}}{\left|c_{j}-z_{0}\right|^{2}}\right\}\|f\|_{[-1,1]}
$$

for every $f \in \mathcal{P}_{n}^{c}\left(a_{1}, a_{2}, \ldots, a_{n} ;[-1,1]\right)$ and $x_{0} \in(-1,1)$, where $z_{0}$ is defined by

$$
z_{0}:=x_{0}+i \sqrt{1-x_{0}^{2}}, \quad x_{0} \in(-1,1) .
$$

Note that

$$
B_{n}\left(x_{0}\right)=\operatorname{Re}\left(\sum_{j=1}^{n} \frac{\sqrt{a_{j}^{2}-1}}{a_{j}-x_{0}}\right)=\sum_{j=1}^{n} \frac{1-\left|c_{j}\right|^{2}}{\left|c_{j}-z_{0}\right|^{2}}, \quad x_{0} \in(-1,1) .
$$


Our next result extends an inequality established by Russak [118 to wider families of rational functions.

Theorem 11.4. Let $\left\{a_{1}, a_{2}, \ldots, a_{n}\right\} \subset \mathbb{C} \backslash \mathbb{R}$. Then

$$
\left|f^{\prime}\left(x_{0}\right)\right| \leq \max \left\{\sum_{\substack{j=1 \\ \operatorname{Im}\left(a_{j}\right)>0}}^{n} \frac{2\left|\operatorname{Im}\left(a_{j}\right)\right|}{\left|x_{0}-a_{j}\right|^{2}}, \sum_{\substack{j=1 \\ \operatorname{Im}\left(a_{j}\right)<0}}^{n} \frac{2\left|\operatorname{Im}\left(a_{j}\right)\right|}{\left|x_{0}-a_{j}\right|^{2}}\right\}\|f\|_{\mathbb{R}}
$$

for every $f \in \mathcal{P}_{n}^{c}\left(a_{1}, a_{2}, \ldots, a_{n} ; \mathbb{R}\right)$ and $x_{0} \in \mathbb{R}$. If the first sum is not less than the second sum for a fixed $x_{0} \in \mathbb{R}$, then equality occurs for $f=c \tilde{S}_{n}^{+}, c \in \mathbb{C}$, where $\tilde{S}_{n}^{+}$is the Blaschke product associated with the poles $a_{j}$ lying in the upper half-plane

$$
H^{+}:=\{z \in \mathbb{C}: \operatorname{Im}(z)>0\} .
$$

If the first sum is not greater than the second sum for a fixed $x_{0} \in \mathbb{R}$, then equality occurs for $f=c \tilde{S}_{n}^{-}, c \in \mathbb{C}$, where $\tilde{S}_{n}^{-}$is the Blaschke product associated with the poles $a_{j}$ lying in the lower half-plane

$$
H^{-}:=\{z \in \mathbb{C}: \operatorname{Im}(z)<0\} .
$$

Our last result in [19] is a Bernstein-Szegö-type inequality for $\mathcal{P}_{n}^{r}\left(a_{1}, a_{2}, \ldots, a_{2 n} ; \mathbb{R}\right)$. It follows from the Bernstein-Szegö-type inequality (11.2) for $\mathcal{P}_{n}^{r}\left(a_{1}, a_{2}, \ldots, a_{n} ;[-1,1]\right)$.

Theorem 11.5. Let

$$
\left\{a_{1}, a_{2}, \ldots, a_{n}\right\} \subset \mathbb{C} \backslash \mathbb{R}, \quad \operatorname{Im}\left(a_{j}\right)>0, \quad j=1,2, \ldots, n .
$$

Then

$$
f^{\prime}\left(x_{0}\right)^{2}+\widehat{B}_{n}\left(x_{0}\right)^{2} f\left(x_{0}\right)^{2} \leq \widehat{B}_{n}\left(x_{0}\right)^{2}\|f\|_{\mathbb{R}}^{2}, \quad x_{0} \in \mathbb{R},
$$

for every $f \in \mathcal{P}_{n}^{r}\left(a_{1}, a_{2}, \ldots, a_{n} ; \mathbb{R}\right)$, where

$$
\widehat{B}_{n}(x):=\sum_{j=1}^{n} \frac{\operatorname{Im}\left(a_{j}\right)}{\left|x-a_{j}\right|^{2}}, \quad x \in \mathbb{R} .
$$

We remark that equality occurs in Theorem 11.5 if and only if $x_{0}$ is a maximum point of $f$ (i.e., $f\left(x_{0}\right)= \pm\|f\|_{\mathbb{R}}$ ) or $f$ is a "Chebyshev polynomial" for the space $\mathcal{P}_{n}^{r}\left(a_{1}, a_{2}, \ldots, a_{n} ; \mathbb{R}\right)$, which can be expressed explicitly by using the results of [36] and [17.

Note that Bernstein's classical inequalities are contained in Theorems 11.1, 11.2, and 11.3 as limiting cases, by taking

$$
\left\{a_{1}^{(k)}, a_{2}^{(k)}, \ldots, a_{n}^{(k)}\right\} \subset \mathbb{C} \backslash D
$$

in Theorems 11.1 and 11.3 so that $\lim _{k \rightarrow \infty}\left|a_{j}^{(k)}\right|=\infty$ for each $j=1,2, \ldots, n$, and by taking

$$
\left\{a_{1}^{(k)}, a_{2}^{(k)}, \ldots, a_{2 n}^{(k)}\right\} \subset \mathbb{C} \backslash \mathbb{R}
$$

in Theorem 11.2 so that $a_{n+j}^{(k)}=\bar{a}_{j}^{(k)}$ and $\lim _{k \rightarrow \infty}\left|\operatorname{Im}\left(a_{j}^{(k)}\right)\right|=\infty$ for each $j=1,2, \ldots, n$. Further results can be obtained as limiting cases by fixing $a_{1}, a_{2}, \ldots, a_{m}, 1 \leq m \leq n$, in Theorems 11.1 and 11.3, and by taking

$$
\left\{a_{1}, a_{2}, \ldots, a_{m}, a_{m+1}^{(k)}, a_{m+2}^{(k)}, \ldots, a_{n}^{(k)}\right\} \subset \mathbb{C} \backslash D
$$

so that $\lim _{k \rightarrow \infty}\left|a_{j}^{(k)}\right|=\infty$ for each $j=m+1, m+2, \ldots, n$. One may also fix the poles $a_{1}, a_{2}, \ldots, a_{m}, a_{n+1}, a_{n+2}, \ldots, a_{n+m}, 1 \leq m \leq n$, in Theorem 11.2 and take

$$
\left\{a_{1}, \ldots, a_{m}, a_{m+1}^{(k)}, \ldots, a_{n}^{(k)}, a_{n+1}, \ldots, a_{n+m}, a_{n+m+1}^{(k)}, \ldots, a_{2 n}^{(k)}\right\} \subset \mathbb{C} \backslash \mathbb{R}
$$

so that $a_{n+j}^{(k)}=\bar{a}_{j}^{(k)}$ and $\lim _{k \rightarrow \infty}\left|\operatorname{Im}\left(a_{j}^{(k)}\right)\right|=\infty$ for each $j=m+1, m+2, \ldots, n$. 


\section{§12. NIKOLSKIĬ-TYPE INEQUALITIES FOR SHIFT-INVARIANT FUNCTION SPACES}

The well-known results of Nikolskiu assert that the essentially sharp inequality

$$
\left\|h_{n}\right\|_{L_{q}[-1,1]} \leq c(p, q) n^{2 / p-2 / q}\left\|h_{n}\right\|_{L_{p}[-1,1]}
$$

holds for all algebraic polynomials $h_{n}$ of degree at most $n$ with complex coefficients and for all $0<p<q \leq \infty$, while the essentially sharp inequality

$$
\left\|t_{n}\right\|_{L_{q}[-\pi, \pi]} \leq c(p, q) n^{1 / p-1 / q}\left\|t_{n}\right\|_{L_{p}[-\pi, \pi]}
$$

holds for all trigonometric polynomials $t_{n}$ of degree at most $n$ with complex coefficients and for all $0<p<q \leq \infty$. The subject started with two famous papers, [116] and [134]. There are quite a few related papers in the literature.

Let $V_{n}$ be a vector space of complex-valued functions defined on $\mathbb{R}$ of dimension $n+1$ over $\mathbb{C}$. We say that $V_{n}$ is shift invariant (on $\mathbb{R}$ ) if $f \in V_{n}$ implies that $f_{a} \in V_{n}$ for every $a \in \mathbb{R}$, where $f_{a}(x):=f(x-a)$ on $\mathbb{R}$. Let $\Lambda_{n}:=\left\{\lambda_{0}, \lambda_{1}, \ldots, \lambda_{n}\right\}$ be a set of distinct complex numbers. The collection of all linear combinations of $e^{\lambda_{0} t}, e^{\lambda_{1} t}, \ldots, e^{\lambda_{n} t}$ over $\mathbb{C}$ will be denoted by

$$
E\left(\Lambda_{n}\right):=\operatorname{span}\left\{e^{\lambda_{0} t}, e^{\lambda_{1} t}, \ldots, e^{\lambda_{n} t}\right\}
$$

The elements of $E\left(\Lambda_{n}\right)$ are called exponential sums of $n+1$ terms. Examples of shift invariant spaces of dimension $n+1$ include $E\left(\Lambda_{n}\right)$. In [66], Theorem 8.5 was proved. Using the $L_{\infty}$ norm on a fixed subinterval $[a+\delta, b-\delta] \subset[a, b]$ in the numerator in Theorem 8.5, in [27] we proved the following essentially sharp result.

Theorem 12.1. If $\Lambda_{n}:=\left\{\lambda_{0}, \lambda_{1}, \ldots, \lambda_{n}\right\}$ is a set of distinct real numbers, then

$$
\|f\|_{L_{\infty}[a+\delta, b-\delta]} \leq e 8^{1 / p}\left(\frac{n+1}{\delta}\right)^{1 / p}\|f\|_{L_{p}[a, b]}
$$

for every $f \in E\left(\Lambda_{n}\right), p>0$, and $\delta \in\left(0, \frac{1}{2}(b-a)\right)$.

The key to this result is the Remez-type inequality of Theorem $2.3^{*}$. Having real exponents $\lambda_{j}$ in the above theorems is essential in the proof, which involves some Descartes system methods. In 32, we proved an analogous result for complex exponents $\lambda_{j}$, in which case Descartes system methods cannot help.

Theorem 12.2. Let $V_{n} \subset C[a, b]$ be a shift invariant vector space of complex-valued functions defined on $\mathbb{R}$ of dimension $n+1$ over $\mathbb{C}$. Let $p \in(0,2]$. Then

$$
\|f\|_{L_{\infty}[a+\delta, b-\delta]} \leq 2^{2 / p^{2}}\left(\frac{n+1}{\delta}\right)^{1 / p}\|f\|_{L_{p}[a, b]}
$$

for every $f \in V_{n}$ and $\delta \in\left(0, \frac{1}{2}(b-a)\right)$.

Problem 12.3. Is it possible to extend a version of Theorem 12.2 to ALL $p>0$ ?

\section{§13. InVERSE MARKOV- AND BERnstein-TyPE INEQUALITIES}

Let $\varepsilon \in[0,1]$, and let $D_{\varepsilon}$ be the ellipse of the complex plane with large axis $[-1,1]$ and small axis $[-i \varepsilon, i \varepsilon]$. Let $\mathcal{P}_{n}^{c}\left(D_{\varepsilon}\right)$ denote the collection of all polynomials of degree $n$ with complex coefficients and with all their zeros in $D_{\varepsilon}$. Extending a result of Turán [136], Eröd [81, III. tétel] proved that

$$
c_{1}(n \varepsilon+\sqrt{n}) \leq \inf _{p} \frac{\left\|p^{\prime}\right\|_{D_{\varepsilon}}}{\|p\|_{D_{\varepsilon}}} \leq c_{2}(n \varepsilon+\sqrt{n}),
$$

where the infimum is taken for all $p \in \mathcal{P}_{n}^{c}\left(D_{\varepsilon}\right)$. Recently Levenberg and Poletsky 95] rediscovered this beautiful result. 
Let $\varepsilon \in[0,1]$, and let $S_{\varepsilon}$ be the diamond of the complex plane with diagonals $[-1,1]$ and $[-i \varepsilon, i \varepsilon]$. Let $\mathcal{P}_{n}^{c}\left(S_{\varepsilon}\right)$ denote the collection of all polynomials of degree $n$ with complex coefficients and with all their zeros in $S_{\varepsilon}$. Let

$$
\|f\|_{A}:=\sup _{z \in A}|f(z)|
$$

for complex-valued functions defined on $A$.

The following result was proved in 69.

Theorem 13.1. There are absolute constants $c_{1}>0$ and $c_{2}>0$ such that

$$
c_{1}(n \varepsilon+\sqrt{n}) \leq \inf _{p} \frac{\left\|p^{\prime}\right\|_{S_{\varepsilon}}}{\|p\|_{S_{\varepsilon}}} \leq c_{2}(n \varepsilon+\sqrt{n}),
$$

where the infimum is taken for all $p \in \mathcal{P}_{n}^{c}\left(S_{\varepsilon}\right)$ with the property

$$
|p(z)|=|p(-z)|, \quad z \in \mathbb{C},
$$

or where the infimum is taken for all real $p \in \mathcal{P}_{n}^{c}\left(S_{\varepsilon}\right)$.

It is an interesting question whether or not the lower bound in Theorem 13.1 holds for all $p \in \mathcal{P}_{n}^{c}(\varepsilon)$. As our next result in [69] shows, this is the case at least when $\varepsilon=1$.

Theorem 13.2. There are absolute constants $c_{1}>0$ and $c_{2}>0$ such that

$$
c_{1} n \leq \inf _{p} \frac{\left\|p^{\prime}\right\|_{S_{1}}}{\|p\|_{S_{1}}} \leq c_{2} n,
$$

where the infimum is taken over all (complex) $p \in \mathcal{P}_{n}^{c}\left(S_{1}\right)$.

Motivated by the author's initial results in this section, Sz. Révész 123 established the right order Turán-type converse Markov inequalities on convex domains of the complex plane. His main theorem contains the results in this section as special cases. Révész's proof is also elementary, but rather subtle.

Theorem 13.3. Let $K \subset \mathbb{C}$ be a bounded convex domain. Then for every $p \in \mathcal{P}_{n}^{c}$ with no zeros in $K$ we have

$$
\frac{\left\|p^{\prime}\right\|_{K}}{\|p\|_{K}} \geq c(K) n, \quad c(K)=0.0003 \frac{w(K)}{d(K)^{2}},
$$

where $d(K)$ is the diameter of $K$ and

$$
w(K):=\min _{\gamma \in[-\pi, \pi]}\left(\max _{z \in K} \operatorname{Re}\left(z e^{-i \gamma}\right)-\min _{z \in K} \operatorname{Re}\left(z e^{-i \gamma}\right)\right)
$$

is the minimal width of $K$.

In particular, the lower bound in Theorem 13.1 holds for all $p \in \mathcal{P}_{n}^{c}(\varepsilon)$.

\section{§14. UltRAFLAT SEQUENCES OF UNIMODULAR POLYNOMIALS}

Let $D$ be the open unit disk of the complex plane. Its boundary, the unit circle of the complex plane, is denoted by $\partial D$. The class

$$
\mathcal{K}_{n}:=\left\{p_{n}: p_{n}(z)=\sum_{k=0}^{n} a_{k} z^{k}, a_{k} \in \mathbb{C},\left|a_{k}\right|=1\right\}
$$

is often called the collection of all (complex) unimodular polynomials of degree $n$. The class

$$
\mathcal{L}_{n}:=\left\{p_{n}: p_{n}(z)=\sum_{k=0}^{n} a_{k} z^{k}, a_{k} \in\{-1,1\}\right\}
$$


is often called the collection of all (real) unimodular polynomials of degree $n$. By Parseval's formula,

$$
\int_{0}^{2 \pi}\left|P_{n}\left(e^{i t}\right)\right|^{2} d t=2 \pi \sum_{k=0}^{n}\left|a_{k}\right|^{2}=2 \pi(n+1)
$$

for all $P_{n} \in \mathcal{K}_{n}$. Therefore,

$$
\min _{z \in \partial D}\left|P_{n}(z)\right| \leq \sqrt{n+1} \leq \max _{z \in \partial D}\left|P_{n}(z)\right| .
$$

An old problem (or rather an old theme) is the following.

Problem 14.1 (Littlewood's flatness problem). How close can a polynomial $P_{n} \in \mathcal{K}_{n}$ or $P_{n} \in \mathcal{L}_{n}$ come to satisfying

$$
\left|P_{n}(z)\right|=\sqrt{n+1}, \quad z \in \partial D ?
$$

Obviously (14.1) is impossible if $n \geq 1$. So one must look for less than (14.1), but then there are various ways of seeking such an "approximate situation". One way is the following. In his paper 98, Littlewood suggested that, conceivably, there might exist a sequence $\left(P_{n}\right)$ of polynomials $P_{n} \in \mathcal{K}_{n}$ (possibly even $P_{n} \in \mathcal{L}_{n}$ ) such that the functions

$$
(n+1)^{-1 / 2}\left|P_{n}\left(e^{i t}\right)\right|
$$

converge to 1 uniformly in $t \in \mathbb{R}$. We shall call such sequences of unimodular polynomials "ultraflat". More precisely, we give the following definition.

Definition 14.2. Given a positive number $\varepsilon$, we say that a polynomial $P_{n} \in \mathcal{K}_{n}$ is $\varepsilon$-flat if

$$
(1-\varepsilon) \sqrt{n+1} \leq\left|P_{n}(z)\right| \leq(1+\varepsilon) \sqrt{n+1}, \quad z \in \partial D .
$$

Definition 14.2* Given a sequence $\left(\varepsilon_{n_{k}}\right)$ of positive numbers tending to 0 , we say that a sequence $\left(P_{n_{k}}\right)$ of polynomials $P_{n_{k}} \in \mathcal{K}_{n_{k}}$ is $\left(\varepsilon_{n_{k}}\right)$-ultraflat if each $P_{n_{k}}$ is $\left(\varepsilon_{n_{k}}\right)$ flat. We simply say that a sequence $\left(P_{n_{k}}\right)$ of polynomials $P_{n_{k}} \in \mathcal{K}_{n_{k}}$ is ultraflat if it is $\left(\varepsilon_{n_{k}}\right)$-ultraflat with a suitable sequence $\left(\varepsilon_{n_{k}}\right)$ of positive numbers tending to 0 .

The existence of ultraflat sequences of unimodular polynomials seemed very unlikely in view of a 1957 conjecture of P. Erdős (Problem 22 in [79]) asserting that, for all $P_{n} \in \mathcal{K}_{n}$ with $n \geq 1$,

$$
\max _{z \in \partial D}\left|P_{n}(z)\right| \geq(1+\varepsilon) \sqrt{n+1},
$$

where $\varepsilon>0$ is an absolute constant (independent of $n$ ). Yet, refining a method of Körner [91, Kahane [90] proved that there exists a sequence $\left(P_{n}\right)$ with $P_{n} \in \mathcal{K}_{n}$ which is $\left(\varepsilon_{n}\right)$-ultraflat, where

$$
\varepsilon_{n}=O\left(n^{-1 / 17} \sqrt{\log n}\right) .
$$

(Kahane's paper contained a slight error though, which was corrected in [125].) Thus, the Erdös conjecture (14.2) was disproved for the classes $\mathcal{K}_{n}$. For the more restricted class $\mathcal{L}_{n}$ the analogous Erdős conjecture is unsettled to this date. It is a common belief that the analogous Erdős conjecture for $\mathcal{L}_{n}$ is true, and consequently there is no ultraflat sequence of polynomials $P_{n} \in \mathcal{L}_{n}$. An interesting result related to Kahane's breakthrough was given by Beck [5]. For an account of some of the work done till the mid 1960s, see Littlewood's book [99] and [125].

Let $\left(\varepsilon_{n}\right)$ be a sequence of positive numbers tending to 0 . Let a sequence $\left(P_{n}\right)$ of polynomials $P_{n} \in \mathcal{K}_{n}$ be $\left(\varepsilon_{n}\right)$-ultraflat. We write

$$
P_{n}\left(e^{i t}\right)=R_{n}(t) e^{i \alpha_{n}(t)}, \quad R_{n}(t)=\left|P_{n}\left(e^{i t}\right)\right|, \quad t \in \mathbb{R} .
$$


It is a simple exercise to show that $\alpha_{n}$ can be chosen so that it is differentiable on $\mathbb{R}$. This property of $\alpha_{n}$ is assumed in the rest of this section. The structure of ultraflat sequences of unimodular polynomials was studied in [56, 58, 59], and [60], where several conjectures of Saffari [126] (see also [124] and [125]) were proved.

Theorem 14.3 (Uniform distribution theorem for the angular speed). Suppose $\left(P_{n}\right)$ is an ultraflat sequence of polynomials $P_{n} \in \mathcal{K}_{n}$. Then, with the notation (14.3), in the interval $[0,2 \pi]$ the distribution of the normalized angular speed $\alpha_{n}^{\prime}(t) / n$ converges to the uniform distribution as $n \rightarrow \infty$. More precisely, we have

$$
m\left(\left\{t \in[0,2 \pi]: 0 \leq \alpha_{n}^{\prime}(t) \leq n x\right\}\right)=2 \pi x+\gamma_{n}(x)
$$

for every $x \in[0,1]$, where

$$
\lim _{n \rightarrow \infty} \max _{x \in[0,1]}\left|\gamma_{n}(x)\right|=0 .
$$

Theorem 14.4 (Negligibility theorem for the higher derivatives). Suppose $\left(P_{n}\right)$ is an ultraflat sequence of polynomials $P_{n} \in \mathcal{K}_{n}$. Then, with the notation (14.3), for every integer $r \geq 2$ we have

$$
\max _{0 \leq t \leq 2 \pi}\left|\alpha_{n}^{(r)}(t)\right| \leq \gamma_{n, r} n^{r}
$$

with suitable constants $\gamma_{n, r}>0$ converging to 0 for every fixed $r=2,3, \ldots$

Theorem 14.5 (The moments of the angular speed). Let $q>0$. Suppose $\left(P_{n}\right)$ is an ultraflat sequence of polynomials $P_{n} \in \mathcal{K}_{n}$. Then we have

$$
\frac{1}{2 \pi} \int_{0}^{2 \pi}\left|\alpha_{n}^{\prime}(t)\right|^{q} d t=\frac{n^{q}}{q+1}+\delta_{n, q} n^{q},
$$

and as a limit case,

$$
\max _{0 \leq t \leq 2 \pi}\left|\alpha_{n}^{\prime}(t)\right|=n+\delta_{n} n,
$$

with suitable constants $\delta_{n, q}$ and $\delta_{n}$ converging to 0 as $n \rightarrow \infty$ for every fixed $q$.

An immediate consequence of Theorem 14.5 is the remarkable fact that for large values of $n \in \mathbb{N}$, the $L_{q}(\partial D)$ Bernstein factors

$$
\frac{\int_{0}^{2 \pi}\left|P_{n}^{\prime}\left(e^{i t}\right)\right|^{q} d t}{\int_{0}^{2 \pi}\left|P_{n}\left(e^{i t}\right)\right|^{q} d t}
$$

of the elements of ultraflat sequences of polynomials $P_{n} \in \mathcal{K}_{n}$ are essentially independent of the polynomials. More precisely, Theorem 14.5 and (14.3) imply the following result.

Theorem 14.6 (The Bernstein factors). Let $\left(P_{n}\right)$ be an $\varepsilon_{n}$-ultraflat sequence of polynomials $P_{n} \in \mathcal{K}_{n}$. For $q>0$ we have

$$
\frac{\int_{0}^{2 \pi}\left|P_{n}^{\prime}\left(e^{i t}\right)\right|^{q} d t}{\int_{0}^{2 \pi}\left|P_{n}\left(e^{i t}\right)\right|^{q} d t}=\frac{n^{q}}{q+1}+o_{n, q} n^{q},
$$

and as a limit case,

$$
\frac{\max _{0 \leq t \leq 2 \pi}\left|P_{n}^{\prime}\left(e^{i t}\right)\right|}{\max _{0 \leq t \leq 2 \pi}\left|P_{n}\left(e^{i t}\right)\right|}=n+o_{n} n,
$$

with suitable constants $o_{n, q}$ and $o_{n}$ converging to 0 as $n \rightarrow \infty$ for every fixed $q$.

In [59], an extension of Saffari's uniform distribution conjecture to higher derivatives was also proved. 
Theorem 14.7. Suppose $\left(P_{n}\right)$ is an $\varepsilon_{n}$-ultraflat sequence of polynomials $P_{n} \in \mathcal{K}_{n}$. Then

$$
m\left\{t \in[0,2 \pi]:\left|P_{n}^{(r)}\left(e^{i t}\right)\right| \leq n^{r+1 / 2} x^{r}\right\}=2 \pi x+o_{r, n}(x)
$$

for every $x \in[0,1]$, where $\lim _{n \rightarrow \infty} o_{r, n}(x)=0$ for every fixed $r=1,2, \ldots$ and $x \in[0,1]$.

For every fixed $r=1,2, \ldots$, the convergence of $o_{n, r}(x)$ is uniform on $[0,1]$ by Dini's Theorem.

For continuous functions $f$ defined on $[0,2 \pi]$, and for $q \in(0, \infty)$, we define

$$
\|f\|_{q}:=\left(\int_{0}^{2 \pi}|f(t)|^{q} d t\right)^{1 / q} \text {. }
$$

We also define

$$
\|f\|_{\infty}:=\lim _{q \rightarrow \infty}\|f\|_{q}=\max _{t \in[0,2 \pi]}|f(t)| .
$$

In [63, based on the results in [59], we resolved yet another conjecture of Saffari and Queffélec; see (1.30) in [125.

Theorem 14.8. Let $q \in(0, \infty)$. If $\left(P_{n}\right)$ is an ultraflat sequence of polynomials $P_{n} \in \mathcal{K}_{n}$, and $q \in(0, \infty)$, then for $f_{n}(t):=\operatorname{Re}\left(P_{n}\left(e^{i t}\right)\right)$ we have

$$
\left\|f_{n}\right\|_{q} \sim\left(\frac{\Gamma\left(\frac{q+1}{2}\right)}{\Gamma\left(\frac{q}{2}+1\right) \sqrt{\pi}}\right)^{1 / q} n^{1 / 2}
$$

and

$$
\left\|f_{n}^{\prime}\right\|_{q} \sim\left(\frac{\Gamma\left(\frac{q+1}{2}\right)}{(q+1) \Gamma\left(\frac{q}{2}+1\right) \sqrt{\pi}}\right)^{1 / q} n^{3 / 2},
$$

where $\Gamma$ denotes the usual gamma function, and the $\sim$ symbol means that the ratio of the left-hand and right-hand sides converges to 1 as $n \rightarrow \infty$.

In [60], we proved Saffari's "near-orthogonality conjecture" below.

Theorem 14.9. Assume that $\left(P_{n}\right)$ is an ultraflat sequence of polynomials $P_{n} \in \mathcal{K}_{n}$. Then

$$
\sum_{k=0}^{n} a_{k, n} a_{n-k, n}=o(n), \quad P_{n}(z):=\sum_{k=0}^{n} a_{k, n} z^{k} .
$$

Here, as usual, o(n) denotes a quantity for which $\lim _{n \rightarrow \infty} o(n) / n=0$. The statement remains true if the ultraflat sequence $\left(P_{n}\right)$ of polynomials $P_{n} \in \mathcal{K}_{n}$ is replaced by an ultraflat sequence $\left(P_{n_{k}}\right)$ of polynomials $P_{n_{k}} \in \mathcal{K}_{n_{k}}, 0<n_{1}<n_{2}<\cdots$.

If $Q_{n}$ is a polynomial of degree $n$ of the form

$$
Q_{n}(z)=\sum_{k=0}^{n} a_{k} z^{k}, \quad a_{k} \in \mathbb{C},
$$

then its conjugate polynomial is defined by

$$
Q_{n}^{*}(z):=z^{n} \bar{Q}_{n}(1 / z):=\sum_{k=0}^{n} \bar{a}_{n-k} z^{k} .
$$

In terms of the above definition, Theorem 14.9 may be rewritten as follows.

Theorem 14.10. Assume that $\left(P_{n}\right)$ is an ultraflat sequence of polynomials $P_{n} \in \mathcal{K}_{n}$. Then

$$
\frac{1}{2 \pi} \int_{\partial D}\left|P_{n}(z)-P_{n}^{*}(z)\right|^{2}|d z|=2 n+o(n) .
$$


We remark that straightforward modifications in the proofs of each of the above theorems show that they remain true if the ultraflat sequence $\left(P_{n}\right)$ of polynomials $P_{n} \in \mathcal{K}_{n}$ is replaced by an ultraflat sequence $\left(P_{n_{k}}\right)$ of polynomials

$$
P_{n_{k}} \in \mathcal{K}_{n_{k}}, \quad 0<n_{1}<n_{2}<\cdots .
$$

In [31, Theorem 3], the following inequality was observed.

Theorem 14.11. Let $P$ be a conjugate reciprocal unimodular polynomial of degree $n$. Then

$$
\max _{z \in \partial D}|P(z)| \geq(1+\varepsilon) \sqrt{n+1}
$$

with $\varepsilon:=\sqrt{4 / 3}-1$.

In [126], another "near orthogonality" relation was conjectured. Namely, it was suspected that if $\left(P_{n_{m}}\right)$ is an ultraflat sequence of polynomials $P_{n_{m}} \in \mathcal{K}_{n_{m}}$, then

$$
\sum_{k=0}^{n} a_{k, n} \bar{a}_{n-k, n}=o(n), \quad P_{n}(z):=\sum_{k=0}^{n} a_{k, n} z^{k}, \quad n=n_{m}, \quad m=1,2, \ldots .
$$

However, it was Saffari himself who showed with Queffélec [125] that this could not be any farther away from being true. They constructed an ultraflat sequence $\left(P_{n_{m}}\right)$ of plain-reciprocal polynomials $P_{n_{m}} \in \mathcal{K}_{n_{m}}$ such that

$$
P_{n}(z):=\sum_{k=0}^{n} a_{k, n} z^{k}, \quad a_{k, n}=a_{n-k, n}, \quad k=0,1,2, \ldots, n,
$$

and hence

$$
\sum_{k=0}^{n} a_{k, n} \bar{a}_{n-k, n}=n+1, \quad n=n_{m}, \quad m=1,2, \ldots
$$

\section{§15. ZEROS OF POLYNOMIALS WITH RESTRICTED COEFFICIENTS}

Let $0 \leq n_{1}<n_{2}<\cdots<n_{N}$ be integers. A cosine polynomial of the form $T_{N}(\theta)=$ $\sum_{j=1}^{N} \cos \left(n_{j} \theta\right)$ must have at least one real zero in a period. This is obvious if $n_{1} \neq 0$, since then the integral of the sum on a period is 0 . The above statement is less obvious if $n_{1}=0$, but for sufficiently large $N$, it follows simply from Littlewood's conjecture. Here we mean Littlewood's already mentioned conjecture with a textbook proof in [44. It is not difficult to prove the statement in general even in the case where $n_{1}=0$. One possible way is to use the identity

$$
\sum_{j=1}^{n_{N}} T_{N}\left((2 j-1) \pi / n_{N}\right)=0 .
$$

See 94, for example. Another way is to use Theorem 2 of [108]. So there is certainly no shortage of possible approaches to prove the starting observation of this section even for $n_{1}=0$.

It seems likely that the number of zeros of the above sums in a period must tend to $\infty$ with $N$. In a private communication, B. Conrey asked how fast the number of zeros of the above sums in a period tends to $\infty$ as a function of $N$. In 43, the authors observed that for an odd prime $p$ the Fekete polynomial $f_{p}(z)=\sum_{k=0}^{p-1}\left(\frac{k}{p}\right) z^{k}$ (the coefficients are Legendre symbols) has $\sim \kappa_{0} p$ zeros on the unit circle, where $0.500813>\kappa_{0}>0.500668$. Conrey's question in general does not appear to be easy.

Littlewood in his 1968 monograph 99, problem 22] posed the following research problem, which appears to be still open: "If the $n_{m}$ are integral and all different, what is the lower bound on the number of real zeros of $\sum_{m=1}^{N} \cos \left(n_{m} \theta\right)$ ? Possibly $N-1$, or not 
much less". Here the real zeros are counted in a period. In fact no progress appears to have been made on this in the last half century. In a recent paper 38. we showed that this is false. There exist cosine polynomials $\sum_{m=1}^{N} \cos \left(n_{m} \theta\right)$ with the $n_{m}$ integral and all different so that the number of their real zeros in the period is $O\left(N^{5 / 6} \log N\right)$ (here the frequencies $n_{m}=n_{m}(N)$ may vary with $N$ ). However, there are reasons to believe that a cosine polynomial $\sum_{m=1}^{N} \cos \left(n_{m} \theta\right)$ always has many zeros in the period. In [33, we proved the following.

Theorem 15.1. Suppose the set $\left\{a_{j}: j \in \mathbb{N}\right\} \subset \mathbb{R}$ is finite and the set $\left\{j \in \mathbb{N}: a_{j} \neq 0\right\}$ is infinite. Let

$$
T_{n}(t)=\sum_{j=0}^{n} a_{j} \cos (j t) .
$$

Then $\lim _{n \rightarrow \infty} \mathcal{N}\left(T_{n}\right)=\infty$, where $\mathcal{N}\left(T_{n}\right)$ denotes the number of sign changes of $T_{n}$ in the period $[-\pi, \pi)$.

The book [13] deals with a number of related topics. Littlewood [96- 100 ] was interested in many closely related problems. See also 31 .

The study of the location of zeros of polynomials from

$$
\mathcal{F}_{n}:=\left\{p: p(x)=\sum_{i=0}^{n} a_{i} x^{i}, a_{i} \in\{-1,0,1\}\right\}
$$

begins with Bloch and Pólya [10. They proved that the average number of real zeros of a polynomial in $\mathcal{F}_{n}$ is at most $c \sqrt{n}$. They also proved that a polynomial from $\mathcal{F}_{n}$ cannot have more than

$$
\frac{c n \log \log n}{\log n}
$$

real zeros. This result, which appears to be the first on this subject, shows that polynomials in $\mathcal{F}_{n}$ do not behave like unrestricted polynomials. Schur [129 and, by different methods, Szegö [133] and Erdős and Turán [80] improved the above bound to $c \sqrt{n \log n}$ (see also [17]).

In 34, we gave the right upper bound of $c \sqrt{n}$ for the number of real zeros of polynomials from a large class, namely, the class of all polynomials of the form

$$
p(x)=\sum_{j=0}^{n} a_{j} x^{j}, \quad\left|a_{j}\right| \leq 1, \quad\left|a_{0}\right|=\left|a_{n}\right|=1, \quad a_{j} \in \mathbb{C} .
$$

In [26], we extended this result by proving the following theorems.

Theorem 15.2. Every polynomial $p$ of the form

$$
p(x)=\sum_{j=0}^{n} a_{j} x^{j}, \quad\left|a_{0}\right|=1, \quad\left|a_{j}\right| \leq 1, \quad a_{j} \in \mathbb{C},
$$

has at most $c \sqrt{n}$ zeros inside any polygon with vertices on the unit circle, where the constant $c$ depends only on the polygon.

Theorem 15.3. There is an absolute constant c such that

$$
p(x)=\sum_{j=0}^{n} a_{j} x^{j}, \quad\left|a_{0}\right|=\left|a_{n}\right|=1, \quad\left|a_{j}\right| \leq 1, \quad a_{j} \in \mathbb{C},
$$

has at most $c(n \alpha+\sqrt{n})$ zeros in the strip $\{z \in \mathbb{C}:|\operatorname{Im}(z)| \leq \alpha\}$, and in the sector $\{z \in \mathbb{C}:|\arg (z)| \leq \alpha\}$. 
Theorem 15.4. Let $\alpha \in(0,1)$. Every polynomial $p$ of the form

$$
p(x)=\sum_{j=0}^{n} a_{j} x^{j}, \quad\left|a_{0}\right|=1, \quad\left|a_{j}\right| \leq 1, \quad a_{j} \in \mathbb{C},
$$

has at most $c / \alpha$ zeros inside any polygon with vertices on the circle $\{z \in \mathbb{C}:|z|=1-\alpha\}$, where the constant $c$ depends only on the number of the vertices of the polygon.

The paper [26] containing Theorems 15.2, 15.3, and 15.4 appeared sooner than [34]. The book [17] contains only a few related weaker results. Our Theorem 2.1 in 34 is a simple consequence of Theorem 15.2, and it sharpens and generalizes results of Amoroso [1], Bombieri and Vaaler [11, and Hua [88, who gave upper bounds for the multiplicity of a zero that a polynomial with integral coefficients may have at 1 .

The sharpness of Theorem 15.2 can be seen by the theorem below, proved in [34.

Theorem 15.5. For every $n \in \mathbb{N}$, there exists a polynomial $p_{n}$ of the form given in Theorem 15.2 with real coefficients so that $p_{n}$ has a zero at 1 with multiplicity at least $\lfloor\sqrt{n}\rfloor-1$.

For $0<\alpha \leq n^{-1 / 2}$, the sharpness of Theorem 15.3 is shown by the polynomials

$$
q_{n}(z):=p_{n}(z)+z^{2 n+1} p_{n}\left(z^{-1}\right),
$$

where the $p_{n}$ are the polynomials in Theorem 15.5. Namely, the polynomials $q_{n}$ of degree $2 n+1$ are of the required form with $\lfloor\sqrt{n}\rfloor-1 \geq c(n \alpha+\sqrt{n})$ zeros at 1 . When $n^{-1 / 2} \leq$ $\alpha \leq 1$, the sharpness of Theorem 15.3 is shown by the polynomials $q_{n}(z):=z^{n}-1$.

The next theorem, proved in [26, shows the sharpness of Theorem 15.4.

Theorem 15.6. For every $\alpha \in(0,1)$, there exists a polynomial $p_{n}$ of the form given in Theorem 15.4 with real coefficients so that $p_{n}$ has a zero at $1-\alpha$ with multiplicity at least $\lfloor 1 / \alpha\rfloor-1$. (It can also be arranged that $n \leq 1 / \alpha^{2}+2$.)

Let $D\left(z_{0}, r\right):=\left\{z \in \mathbb{C}:\left|z-z_{0}\right|<r\right\}$ denote the open disk of the complex plane centered at $z_{0} \in \mathbb{C}$ with radius $r>0$. As a remark to Theorem 15.5, we point out that a more or less straightforward application of Jensen's formula gives that there is an absolute constant $c>0$ such that every polynomial $p$ of the form

$$
p(x)=\sum_{j=0}^{n} a_{j} x^{j}, \quad\left|a_{0}\right|=1, \quad\left|a_{j}\right| \leq 1, \quad a_{j} \in \mathbb{C},
$$

has at most $(c / \alpha) \log (1 / \alpha)$ zeros in the disk $D(0,1-\alpha), \alpha \in(0,1)$. An example in [57, involving only real coefficients, suggested by Nazarov, shows that this upper bound for the number of zeros in the disk $D(0,1-\alpha)$ is, up to the absolute constant $c>0$, best possible. So, in particular, the constant in Theorem 15.4 cannot be made independent of the number of vertices of the polygon.

Also, it was shown in [57] that there are polynomials $p \in \mathcal{K}_{n}$ with no zeros in the annulus

$$
\{z \in \mathbb{C}: 1-c \log n / n<|z|<1+c \log n / n\},
$$

where $c$ is an absolute constant. It is conjectured that every $p \in \mathcal{K}_{n}$ has a zero in the annulus $\{z \in \mathbb{C}: 1-c / n<|z|<1+c / n\}$, where $c>0$ is an absolute constant.

The class $\mathcal{F}_{n}$ and various related classes have been studied from several points of view. Littlewood's monograph 99. contains a number of interesting, challenging, and still open problems about polynomials with coefficients from $\{-1,1\}$. The distribution of zeros of polynomials with coefficients from $\{0,1\}$ was studied in [117] by Odlyzko and Poonen. 
Bloch and Pólya [10] also proved that there are polynomials $p \in \mathcal{F}_{n}$ with

$$
\frac{c n^{1 / 4}}{\sqrt{\log n}}
$$

distinct real zeros of odd multiplicity. (Schur [129] claimed they did it for polynomials with coefficients only from $\{-1,1\}$, but this appears to be incorrect.)

In the seminal paper 100, Littlewood and Offord proved that the number of real roots of a $p \in \mathcal{L}_{n}$, on average, lies between

$$
\frac{c_{1} \log n}{\log \log \log n} \text { and } c_{2} \log ^{2} n,
$$

and it was proved by Boyd [0] that every $p \in \mathcal{L}_{n}$ has at most $c \log ^{2} n / \log \log n$ zeros at 1 (in the sense of multiplicity). It is conjectured that every $p \in \mathcal{L}_{n}$ has at most $c \log n$ zeros at 1 , with an absolute constant $c>0$.

Kac 89 showed that the expected number of real roots of a polynomial of degree $n$ with random uniformly distributed coefficients is asymptotically $(2 / \pi) \log n$. He wrote: "I have also stated that the same conclusion holds if the coefficients assume only the values 1 and -1 with equal probabilities. Upon closer examination it turns out that the proof I had in mind is inapplicable... This situation tends to emphasize the particular interest of the discrete case, which surprisingly enough turns out to be the most difficult." In a related paper, Solomyak [132] studied the random series $\sum \pm \lambda^{n}$.

In [71, we improved the lower bound (15.1) in the result of Bloch and Pólya to $c n^{1 / 4}$. Moreover, we allowed a much more general coefficient constraint in our main result. Our approach is quite different from that of Bloch and Pólya.

Theorem 15.7. There are absolute constants $c_{1}>0$ and $c_{2}>0$ such that for every

$$
\left\{a_{0}, a_{1}, \ldots, a_{n}\right\} \subset[1, M], \quad 1 \leq M \leq \exp \left(c_{1} n^{1 / 4}\right),
$$

there are

$$
b_{0}, b_{1}, \ldots, b_{n} \in\{-1,0,1\}
$$

such that

$$
P(z)=\sum_{j=0}^{n} b_{j} a_{j} z^{j}
$$

has at least $c_{2} n^{1 / 4}$ distinct sign changes in $(0,1)$.

Let $\partial D$ denote the unit circle of the complex plane. Let

$$
\|P\|:=\max _{z \in \partial D}|P(z)| .
$$

A classical result of Erdős and Turán [80] is the following.

Theorem (Erdős-Turán). If the zeros of

$$
P(z):=\sum_{j=0}^{n} a_{j} z^{j}, \quad a_{j} \in \mathbb{C}, \quad a_{0} a_{n} \neq 0
$$

are denoted by

$$
z_{j}=r_{j} \exp \left(i \varphi_{j}\right), \quad r_{j}>0, \quad \varphi_{j} \in[0,2 \pi), \quad j=1,2, \ldots, n,
$$

then for every $0 \leq \alpha<\beta \leq 2 \pi$ we have

$$
\left|\sum_{j \in I(\alpha, \beta)} 1-\frac{\beta-\alpha}{2 \pi} n\right|<16 \sqrt{n \log R},
$$


where

and

$$
R:=\left|a_{0} a_{n}\right|^{-1 / 2}\|P\|
$$

$$
I(\alpha, \beta):=\left\{j: \alpha \leq \varphi_{j} \leq \beta\right\} .
$$

Note that some books quote this result with

$$
R:=\left|a_{0} a_{n}\right|^{-1 / 2}\left(\left|a_{0}\right|+\left|a_{1}\right|+\cdots+\left|a_{n}\right|\right)
$$

in place of $R:=\left|a_{0} a_{n}\right|^{-1 / 2}\|P\|$. In fact, the weaker result is an obvious consequence of the stronger one by observing that $\|P\| \leq\left|a_{0}\right|+\left|a_{1}\right|+\cdots+\left|a_{n}\right|$. In [70], we offered a subtle improvement of the above Erdős-Turán theorem.

Theorem 15.8. If the zeros of

$$
P(z):=\sum_{j=0}^{n} a_{j} z^{j}, \quad a_{j} \in \mathbb{C}, \quad a_{0} a_{n} \neq 0,
$$

are denoted by

$$
z_{j}=r_{j} \exp \left(i \varphi_{j}\right), \quad r_{j}>0, \quad \varphi_{j} \in[0,2 \pi), \quad j=1,2, \ldots, n,
$$

then for every $0 \leq \alpha<\beta \leq 2 \pi$ we have

$$
\sum_{j \in I_{1}(\alpha, \beta)} 1-\frac{\beta-\alpha}{2 \pi} n \leq 16 \sqrt{n \log R_{1}}
$$

and

$$
\sum_{j \in I_{2}(\alpha, \beta)} 1-\frac{\beta-\alpha}{2 \pi} n \leq 16 \sqrt{n \log R_{2}},
$$

where

and

$$
R_{1}:=\left|a_{n}\right|^{-1}\|P\|, \quad R_{2}:=\left|a_{0}\right|^{-1}\|P\|,
$$

$$
I_{1}(\alpha, \beta):=\left\{j: \alpha \leq \varphi_{j} \leq \beta, r_{j} \geq 1\right\}, \quad I_{2}(\alpha, \beta):=\left\{j: \alpha \leq \varphi_{j} \leq \beta, r_{j} \leq 1\right\} .
$$

This result is closely related to a recent paper of V. Totik and P. Varjú [135. In fact, it may as well be derived from part (ii) of Theorem 1.1 in [135. However, here we do not rely on this recent result. Our approach is based on the interesting observation that the Erdős-Turán theorem above improves itself.

A straightforward consequence of the above theorem is the following.

Corollary 15.9. If the modulus of a monic polynomial $P$ of degree $n$ (with complex coefficients) on the unit circle of the complex plane is at most $1+o(1)$ uniformly, then the multiplicity of each zero of $P$ on the unit circle is $o\left(n^{1 / 2}\right)$.

\section{REFERENCES}

[1] F. Amoroso, Sur le diamètre transfini entier d'un intervalle réel, Ann. Inst. Fourier (Grenoble) 40 (1990), 885-911 (1991). MR1096596 (92j:11070)

[2] V. V. Arestov, On integral inequalities for trigonometric polynomials and their derivatives, Izv. Akad. Nauk SSSR Ser. Mat. 45 (1981), no. 1, 3-22; English transl. in Math. USSR-Izv. 18 (1981), no. 1. MR0607574 (82c:26019)

[3] J. Bak and D. J. Newman, Rational combinations of $x^{\lambda_{k}}, \lambda_{k} \geq 0$ are always dense in C[0,1], J. Approx. Theory 23 (1978), 155-157. MR0487180 (58:6840)

[4] J. Bastero, $\ell_{q}$-subspaces of stable p-Banach spaces, $0<p \leq 1$, Arch. Math. (Basel) 40 (1983), 538-544. MR:0710019 (84i:46021)

[5] J. Beck, Flat polynomials on the unit circle - note on a problem of Littlewood, Bull. London Math. Soc. 23 (1991), 269-277. MR1123337 (93b:42002) 
[6] D. Benko and T. Erdélyi, Markov inequality for polynomials of degree $n$ with $m$ distinct zeros, J. Approx. Theory 122 (2003), 241-248. MR.1988302 (2004f:41008)

[7] D. Benko, T. Erdélyi, and J. Szabados, The full Markov-Newman inequality for Müntz polynomials on positive intervals, Proc. Amer. Math. Soc. 131 (2003), 2385-2391. MR1974635 (2004f:41009)

[8] S. N. Bernstein, Sur la représentation des polynômes positifs, Soobshcheniya Khar'kov. Mat. Obshch. Ser. 214 (1915), 227-228.

[9] F. Beaucoup, P. Borwein, D. Boyd, and C. Pinner, Multiple roots of $[-1,1]$ power series, J. London Math. Soc. (2) 57 (1998), no. 1, 135-147. MR1624809 (99c:30005)

[10] A. Bloch and G. Pólya, On the roots of certain algebraic equations, Proc. London Math. Soc. (2) 33 (1932), 102-114.

[11] E. Bombieri and J. Vaaler, Polynomials with low height and prescribed vanishing, Analytic Number Theory and Diophantine Problems (Stillwater, OK, 1984), Progr. Math., vol. 70, Birkhäuser Boston, Boston, MA, 1987, pp. 53-73. MR1018369 (90k:11133)

[12] P. Borwein, Markov's inequality for polynomials with real zeros, Proc. Amer. Math. Soc. 93 (1985), 43-47. MR0766524 (86g:41024)

[13] Computational excursions in analysis and number theory, CMS Books in Math. / Ouvrages de Math. de la SMC, vol. 10, Springer, New York, 2002. MR.1912495 (2003m:11045)

[14] P. Borwein and T. Erdélyi, Markov-Bernstein-type inequalities for classes of polynomials with restricted zeros, Constr. Approx. 10 (1994), 411-425. MR.1291056 (95f:41023)

[15] Markov and Bernstein type inequalities in $L_{p}$ for classes of polynomials with constraints, J. London Math. Soc. (2) 51 (1995), 573-588. MR1332893 (96g:41011)

[16] Upper bounds for the derivative of exponential sums, Proc. Amer. Math. Soc. 123 (1995), 1481-1486. MR 1232137 (95f:41024)

[17] , Polynomials and polynomial inequalities, Grad. Texts in Math., vol. 161, Springer-Verlag, New York, NY, 1995. MR.1367960 (97e:41001)

[18] Müntz spaces and Remez inequalities, Bull. Amer. Math. Soc. (N.S.) 32 (1995), 38-42. MR:1273395 (95d:41026)

[19] Sharp extensions of Bernstein's inequality to rational spaces, Mathematika 43 (1996), 413-423 (1997). MR1433285 (97k:26014)

[20] _ The full Müntz theorem in $C[0,1]$ and $L_{1}[0,1]$, J. London Math. Soc. (2) 54 (1996), 102-110. MR:1395070 (97h:41038)

[21] _ Newman's inequality for Müntz polynomials on positive intervals, J. Approx. Theory 85 (1996), 132-139. MR1385812 (97a:41007)

[22] - The $L_{p}$ version of Newman's inequality for lacunary polynomials, Proc. Amer. Math. Soc. 124 (1996), 101-109. MR1285974 (96j:41015)

[23] _ A sharp Bernstein-type inequality for exponential sums, J. Reine Angew. Math. 476 (1996), 127-141. MR1401698 (97e:41026)

[24] (96g:11077)

[25] Generalizations of Müntz's theorem via a Remez-type inequality for Müntz spaces, J. Amer. Math. Soc. 10 (1997), 327-349. MR1415318 (97k:41027)

[26] On the zeros of polynomials with restricted coefficients, Illinois J. Math. 41 (1997), 667675. MR 1468873 (98g:30008)

[27] _ Pointwise Remez- and Nikolskii-type inequalities for exponential sums, Math. Ann. 316 (2000), 39-60. MR:1735078 (2001a:41015)

[28] _ Markov- and Bernstein-type inequalities for polynomials with restricted coefficients, Ramanujan J. 1 (1997), 309-323. MR.1606930 (98m:41021)

[29] Littlewood-type problems on subarcs of the unit circle, Indiana Univ. Math. J. 46 (1997), 1323-1346. MR:1631600 (99j:30004)

[30] _ Markov-Bernstein type inequalities under Littlewood-type coefficient constraints, Indag. Math. (N.S.) 11 (2000), 159-172. MR.1813157(2002a:26019)

[31] L L L Ler bounds for the merit factors of trigonometric polynomials from Littlewood classes, J. Approx. Theory 125 (2003), 190-197. MR2019608 (2004j:41013)

[32] - Nikolskii-type inequalities for shift invariant function spaces, Proc. Amer. Math. Soc. 134 (2006), 3243-3246. MR2231907 (2007e:41014)

[33] L Lower bounds for the number of zeros of cosine polynomials in the period: a problem of Littlewood, Acta Arith. 128 (2007), 377-384. MR2320719 (2008e:41010)

[34] P. Borwein, T. Erdélyi, and G. Kós, Littlewood-type problems on [0,1], Proc. London Math. Soc. (3) 79 (1999), 22-46. MR.1687555 (2000c:11111) 
[35] P. Borwein, T. Erdélyi, and J. Zhang, Müntz systems and orthogonal Müntz-Legendre polynomials, Trans. Amer. Math. Soc. 342 (1994), 523-542. MR1227091 (94f:42026)

[36] _ Chebyshev polynomials and Markov-Bernstein type inequalities for rational spaces, J. London Math. Soc. (2) 50 (1994), 501-519. MR1299454 (95j:41015)

[37] P. Borwein, T. Erdélyi, and F. Littmann, Polynomials with coefficients from a finite set, Trans. Amer. Math. Soc. 360 (2008), no. 10, 5145-5154. MR2415068 (2009d:30005)

[38] P. Borwein, T. Erdélyi, R. Ferguson, and R. Lockhart, On the zeros of cosine polynomials: solution to a problem of Littlewood, Ann. of Math. (2) 167 (2008), 1109-1117. MR2415396 (2009d:42001)

[39] P. Borwein, C. Pinner, and I. Pritsker, Monic integer Chebyshev problem, Math. Comp. 72 (2003), 1901-1916. MR.1986811 (2004e:11022)

[40] D. Boyd, On a problem of Byrnes concerning polynomials with restricted coefficients, Math. Comp. 66 (1997), 1697-1703. MR1433263 (98a:11033)

[41] D. Braess, Nonlinear approximation theory, Springer Ser. Comput. Math., vol. 7, Springer-Verlag, Berlin, 1986. MR0866667 (88e:41002)

[42] J. A. Clarkson and P. Erdős, Approximation by polynomials, Duke Math. J. 10 (1943), 5-11. MR.0007813 (4:196e)

[43] B. Conrey, A. Granville, B. Poonen, and K. Soundararajan, Zeros of Fekete polynomials, Ann. Inst. Fourier (Grenoble) 50 (2000), 865-889. MR.1779897 (2001h:11108)

[44] R. A. DeVore and G. G. Lorentz, Constructive approximation, Grundlehren Math. Wiss., Bd. 303, Springer-Verlag, Berlin, 1993. MR.1261635 (95f:41001)

[45] Z. Ditzian and D. Lubinsky, Jackson and smoothness theorems for Freud weights in $L_{p}(0<p \leq$ $\infty)$, Constr. Approx. 13 (1997), 99-152. MR1424365 (97k:41028)

[46] T. Erdélyi, The Remez inequality on the size of polynomials, Approximation Theory VI, Vol. I (College Station, TX, 1989) (C. K. Chui, L. L. Schumaker, J. D. Wards, eds.), Acad. Press, Boston, MA, 1989, pp. 243-246. MR.1090997 (91k:41025)

[47] _ Markov-type estimates for certain classes of constrained polynomials, Constr. Approx. $\mathbf{5}$ (1989), 347-356. MR0996935 (90f:41009)

[48] _ Estimates for the Lorentz degree of polynomials, J. Approx. Theory 67 (1991), 187-198. MR.1133059 (92m:41023)

[49] , Bernstein-type inequality for the derivatives of constrained polynomials, Proc. Amer. Math. Soc. 112 (1991), 829-838. MR.1036985 (91j:41017)

[50] _ Bernstein and Markov type inequalities for generalized nonnegative polynomials, Canad. J. Math. 43 (1991), 495-505. MR.1118006 (92g:41019)

[51] _ Remez-type inequalities on the size of generalized polynomials, J. London Math. Soc. (2) 45 (1992), 255-264. MR1171553 (93e:41022)

[52] _ Remez-type inequalities and their applications, J. Comput. Appl. Math. 47 (1993), 167209. MR $1237312(94 \mathrm{~m}: 26003)$

[53] _ Markov-Bernstein type inequalities for constrained polynomials with real versus complex coefficients, J. Anal. Math. 74 (1998), 165-181. MR.1631654 (99d:41025)

[54] Markov-type inequalities for constrained polynomials with complex coefficients, Illinois J. Math. 42 (1998), 544-563. MR.1648584 (2000e:41024)

[55] Markov- and Bernstein-type inequalities for Müntz polynomials and exponential sums in $L_{p}$, J. Approx. Theory 104 (2000), 142-152. MR1753516 (2001c:41014)

[56] - The resolution of Saffari's phase problem, C. R. Acad. Sci. Paris Sér. I Math. 331 (2000), no. 10, 803-808. MR 1807192 (2001j:30005)

[57] On the zeros of polynomials with Littlewood-type coefficient constraints, Michigan Math. J. 49 (2001), 97-111. MR.1827077 (2002c:30009)

[58] How far is an ultraflat sequence of unimodular polynomials from being conjugatereciprocal? Michigan Math. J. 49 (2001), 259-264. MR.1852302 (2002g:30005)

[59] - The phase problem of ultraflat unimodular polynomials: the resolution of the conjecture of Saffari, Math. Ann. 321 (2001), 905-924. MR1872534 (2003a:30004)

[60] - Proof of Saffari's near-orthogonality conjecture for ultraflat sequences of unimodular polynomials, C. R. Acad. Sci. Paris Sér. I Math. 333 (2001), no. 7, 623-628. MR.1868226 (2002h:42045)

[61] _ The "full Clarkson-Erdös-Schwartz theorem" on the closure of non-dense Müntz spaces, Studia Math. 155 (2003), 145-152. MR1961190 (2003m:30008)

[62] - Extremal properties of the derivatives of the Newman polynomials, Proc. Amer. Math. Soc. 131 (2003), 3129-3134. MR.1992853 (2004c:41020)

[63] - On the real part of ultraflat sequences of unimodular polynomials: consequences implied by the resolution of the phase problem, Math. Ann. 326 (2003), 489-498. MR.1992274 (2004d:41016) 
[64] ㄴ. The "full Müntz theorem" revisited, Constr. Approx. 21 (2005), 319-335. MR2122313 (2006c:41016)

[65] , Bernstein-type inequalities for linear combinations of shifted Gaussians, Bull. London Math. Soc. 38 (2006), 124-138. MR.2201611 (2006i:41012)

[66] Markov-Nikolskii type inequalities for exponential sums on finite intervals, Adv. Math. 208 (2007), 135-146. MR.2304313 (2008a:41006)

[67] - The Remez inequality for linear combinations of shifted Gaussians, Math. Proc. Cambridge Philos. Soc. 146 (2009), no. 3, 523-530. MR2496341

[68] - Newman's inequality for increasing exponential sums, Number Theory and Polynomials, London Math. Soc. Lecture Note Ser., No. 352, Cambridge Univ. Press, Cambridge, 2008, pp. 127141. MR2428519 (2009i:41012)

[69] - Inequalities for exponential sums via interpolation and Turán-type reverse Markov inequalities, Frontiers in Interpolation and Approximation, Pure Appl. Math. (Boca Raton), vol. 282, Chapman \& Hall/CRC, Boca Raton, FL, 2007, pp. 119-144. MR.2274176 (2007g:41013)

[70] An improvement of the Erdös-Turán theorem on the distribution of zeros of polynomials, C. R. Math. Acad. Sci. Paris 346 (2008), no. 5-6, 267-270. MR2414166 (2009e:30010)

[71] Extensions of the Bloch-Pólya theorem on the number of real zeros of polynomials, J. Théor. Nombres Bordeaux 20 (2008), no. 2, 281-287. MR 2477504(2009i:11033)

[72] T. Erdélyi and W. Johnson, The "full Müntz theorem" in $L_{p}[0,1]$ for $0<p<\infty$, J. Anal. Math. 84 (2001), 145-172. MR.1849200(2002e:41012)

[73] T. Erdélyi and J. Szabados, On polynomials with positive coefficients, J. Approx. Theory 54 (1988), 107-122. MR0951032 (91g:41026)

[74] T. Erdélyi, A. Magnus, and P. Nevai, Generalized Jacobi weights, Christoffel functions, and Jacobi polynomials, SIAM J. Math. Anal. 25 (1994), 602-614. MR.1266580 (95f:33011a)

[75] T. Erdélyi and P. Nevai, Lower bounds for derivatives of polynomials and Remez type inequalities, Trans. Amer. Math. Soc. 349 (1997), 4953-4972. MR1407486 (98c:33007)

[76] T. Erdélyi, A. Máté, and P. Nevai, Inequalities for generalized nonnegative polynomials, Constr. Approx. 8 (1992), 241-255. MR1152881(93e:41020)

[77] T. Erdélyi and J. Szabados, On a generalization of the Bernstein-Markov inequality, Algebra i Analiz 14 (2002), no. 4, 36-53; English transl., St. Petersburg Math. J. 14 (2003), no. 4, 563-576. MR.1935916 (2003k:41012)

[78] P. Erdős, On extremal properties of the derivatives of polynomials, Ann. of Math. (2) 41 (1940), 310-313. MR0001945 (1:323g)

[79] - Some unsolved problems, Michigan Math. J. 4 (1957), 291-300. MR0098702 (20:5157)

[80] P. Erdős and P. Turán, On the distribution of roots of polynomials, Ann. of Math. (2) 51 (1950), 105-119. MR0033372(11:431b)

[81] J. Erőd, Bizonyos polinomok maximumának alsó korlátjáról, Matematikai és Fizikai Lapok 46 (1939), 83-84.

[82] C. Frappier, Quelques problèmes extrémaux pour les polynômes et les fonctions entières de type exponentiel, Ph. D. Dissertation, Univ. Montréal, 1982.

[83] G. Freud, Orthogonal polynomials, Pergamon Press, Oxford, 1971.

[84] C. S. Güntürk, Approximation by power series with \pm 1 coefficients, Int. Math. Res. Not. 2005, no. 26, 1601-1610. MR2148266 (2006c:30040)

[85] G. Halász, Markov-type inequalities for polynomials with restricted zeros, J. Approx. Theory 101 (1999), 148-155. MR:1724030 (2000i:41017)

[86] K. Hare and C. Smyth, The monic integer transfinite diameter, Math. Comp. 75 (2006), 19972019. MR2240646(2007h:11037)

[87] F. Hausdorff, Summationsmethoden und Momentfolgen. I, Math. Z. 9 (1921), 74-109. MR1544453

[88] L. K. Hua, Introduction to number theory, Springer-Verlag, Berlin-New York, 1982. MR0665428 (83f:10001)

[89] M. Kac, On the average number of real roots of a random algebraic equation. II, Proc. London Math. Soc. (2) 50 (1949), 390-408. MR0030713(11:40e)

[90] J. P. Kahane, Sur les polynômes à coefficients unimodulaires, Bull. London Math. Soc. 12 (1980), 321-342. MR0587702 (82a:30003)

[91] T. Körner, On a polynomial of Byrnes, Bull. London Math. Soc. 12 (1980), 219-224. MR0572106 (81i:30009)

[92] S. Konyagin, On a question of Pichorides, C. R. Acad. Sci. Paris Sér I Math. 324 (1997), 385-388. MR:1440953 (97k:42002)

[93] - On the Littlewood problem, Izv. Akad. Nauk SSSR Ser. Mat. 45 (1981), no. 2, 243-265; English transl., Math. USSR-Izv. 18 (1981), no. 2, 205-225 (1982). MR0616222 (83d:10045) 
[94] S. V. Konyagin and V. F. Lev, Character sums in complex half-planes, J. Théor. Nombres Bordeaux 16 (2004), no. 3, 587-606. MR2144960 (2006a:11107)

[95] N. Levenberg and E. Poletsky, Reverse Markov inequality, Ann. Acad. Sci. Fenn. Math. 27 (2002), 173-182. MR.1884358 (2003e:30006)

[96] J. E. Littlewood, On the mean values of certain trigonometric polynomials, J. London Math. Soc. 36 (1961), 307-334. MR0141934 (25:5331a)

[97] - On the real roots of real trigonometrical polynomials. II, J. London Math. Soc. 39 (1964), 511-552. MR0171117 (30:1348)

[98] - On polynomials $\sum \pm z^{m}$ and $\sum e^{\alpha_{m} i} z^{m}, z=e^{\theta i}$, J. London Math. Soc. 41 (1966), 367376. MR0196043 (33:4237)

[99] Some problems in real and complex analysis, D. C. Heath and Co., Lexington, MA, 1968. MR0244463 (39:5777)

[100] J. E. Littlewood and A. C. Offord, On the number of real roots of a random algebraic equation. II, Proc. Cambridge Philos. Soc. 35 (1939), 133-148.

[101] J. Szabados and A. Kroó, Constructive properties of self-reciprocal polynomials, Analysis 14 (1994), 319-339. MR1310618 (95m:41038)

[102] G. G. Lorentz, The degree of approximation by polynomials with positive coefficients, Math. Ann. 151 (1963), 239-251. MR0155135 (27:5075)

[103] _ Notes on approximation, J. Approx. Theory 56 (1989), 360-365. MR0990350 (90f:41004)

[104] Approximation of functions, 2nd ed., Chelsea Publ. Co., New York, 1986. MR0917270 (88j:41001)

[105] G. G. Lorentz, M. von Golitschek, and Y. Makovoz, Constructive approximation. Advanced problems, Grundlehren Math. Wiss., Bd. 304, Springer-Verlag, Berlin, 1996. MR.1393437 (97k:41002)

[106] G. V. Milovanović, D. S. Mitrinović, and Th. M. Rassias, Topics in polynomials: extremal problems, inequalities, zeros, World Sci. Publ. Co., Inc., River Edge, NJ, 1994. MR1298187 (95m:30009)

[107] O. C. McGehee, L. Pigno, and B. Smith, Hardy's inequality and the $L^{1}$ norm of exponential sums, Ann. of Math. (2) 113 (1981), 613-618. MR0621019 (83c:43002b)

[108] I. D. Mercer, Unimodular roots of special Littlewood polynomials, Canad. Math. Bull. 49 (2006), no. 3, 438-447. MR2252265 (2007h:30008)

[109] H. N. Mhaskar, Introduction to the theory of weighted polynomial approximation, Ser. Approx. Decompos., vol. 7, World Publ. Co., Inc., River Edge, NJ, 1996. MR.1469222 (98i:41014)

[110] When is approximation by Gaussian networks necessarily a linear process? Neural Networks 17 (2004), 989-1001.

[111] H. L. Montgomery, Ten lectures on the interface between analytic number theory and harmonic analysis, CBMS Regional Conf. Ser. in Math., vol. 84, Amer. Math. Soc., Providence, RI, 1994. MR.1297543 (96i:11002)

[112] C. Müntz, Uber den Approximationsatz von Weierstrass, H. A. Schwartz Festschrift, Berlin, 1914.

[113] F. L. Nazarov, Local estimates of exponential polynomials and their applications in inequalities of uncertainty principle type, Algebra i Analiz 5 (1993), no. 4, 3-66; English transl., St. Petersburg Math. J. 5 (1994), no. 4, 663-717. MR.1246419 (94k:42004)

[114] D. J. Newman, Derivative bounds for Müntz polynomials, J. Approx. Theory 18 (1976), 360-362. MR.0430604 (55:3609)

[115] _ Approximation with rational functions, CBMS Regional Conf. Ser. in Math., vol. 41, Conf. Board of Math. Sci., Washington, DC, 1979. MR0539314 (84k:41019)

[116] S. M. Nikol'skiı̌, Inequalities for entire functions of finite degree and their application in the theory of differentiable functions of several variables, Trudy Mat. Inst. Steklov. 38 (1951), 244278. (Russian) MR0048565(14:32e)

[117] A. Odlyzko and B. Poonen, Zeros of polynomials with 0,1 coefficients, Enseign. Math. (2) 39 (1993), 317-348. MR1252071 (95b:11026)

[118] P. P. Petrushev and V. A. Popov, Rational approximation of real functions, Encyclopedia Math. Appl., vol. 28, Cambridge Univ. Press, Cambridge, 1987. MR0940242 (89i:41022)

[119] S. K. Pichorides, Notes on trigonometric polynomials, Conference on Harmonic Analysis in Honor of Antoni Zygmund, Vol. I (Chicago, Ill., 1981), Wadsworth Math. Ser., Wadsworth, Belmont, CA, 1983, pp. 84-94. MR0730060 (85d:42001)

[120] Q. I. Rahman and G. Schmeisser, Les inegalités de Markoff et de Bernstein, Sém. Math. Supér., vol. 86, Presses Univ. Montréal, Montreal, QC, 1983. MR0729316 (85f:41009)

[121] _ Analytic theory of polynomials, London Math. Soc. Monogr. New Ser., vol. 26, Oxford Univ. Press, Oxford, 2002. MR1954841 (2004b:30015)

[122] E. J. Remez, Sur une propriété extrémale des polynômes de Tchebyscheff, Zap. Nauchn.-Issled. Inst. Mat. i Mekh. Khar'kov. Univ. i Khar'kov. Mat. Obshch. (4) 13 (1936), vyp. 1, 93-95. 
[123] Sz. Révész, Turán type reverse Markov inequalities for compact convex sets, J. Approx. Theory 141 (2006), 162-173. MR.2252096 (2007f:41013)

[124] H. Queffélec and B. Saffari, Unimodular polynomials and Bernstein's inequalities, C. R. Acad. Sci. Paris Sér. I Math. 321 (1995), 313-318. MR.1346133 (96h:30003)

[125] On Bernstein's inequality and Kahane's ultraflat polynomials, J. Fourier Anal. Appl. 2 (1996), 519-582. MR1423528 (97h:41027)

[126] B. Saffari, The phase behavior of ultraflat unimodular polynomials, Probabilistic and Stochastic Methods in Analysis, with Applications (Il Ciocco, 1991), NATO Adv. Sci. Inst. Ser. C Math. Phys. Sci., vol. 372, Kluwer Acad. Publ., Dordrecht, 1992, pp. 555-572. MR.1187327 (94m:42060)

[127] J. T. Scheick, Inequalities for derivatives of polynomials of special type, J. Approx. Theory 6 (1972), 354-358. MR0342909 (49:7653)

[128] E. Schmidt, Zur Kompaktheit bei Exponentialsummen, J. Approx. Theory 3 (1970), 445-454. MR0271588 (42:6471)

[129] I. Schur, Untersuchungen über algebraische Gleichungen, Sitz. Preuss. Akad. Wiss., Phys.-Math. Kl. 1933, 403-428.

[130] T. Sheil-Small, Complex polynomials, Cambridge Stud. Adv. Math., vol. 75, Cambridge Univ. Press, Cambridge, 2002. MR1962935 (2004b:30001)

[131] G. Somorjai, A Müntz-type problem for rational approximation, Acta Math. Acad. Sci. Hungar. 27 (1976), 197-199. MR0430617 (55:3622)

[132] B. Solomyak, On the random series $\sum \pm \lambda^{n}$ (an Erdös problem), Ann. of Math. (2) 142 (1995), 611-625. MR 1356783 (97d:11125)

[133] G. Szegö, Bemerkungen zu einem Satz von E. Schmidt uber algebraische Gleichungen, Sitz. Preuss. Akad. Wiss., Phys.-Math. Kl. 1934, 86-98.

[134] G. Szegő and A. Zygmund, On certain mean values of polynomials, J. Anal. Math. 3 (1954), 225-244. MR0064910(16:355c)

[135] V. Totik and P. Varjú, Polynomials with prescribed zeros and small norm, Acta Sci. Math. (Szeged) 73 (2007), 593-611. MR2380067 (2009b:30012)

[136] P. Turán, Über die Ableitung von Polynomen, Compositio Math. 7 (1939), 89-95. MR0000228 $(1: 37 \mathrm{~b})$

[137] On a new method of analysis and its applications, Wiley, New York, NY, 1984. MR0749389 (86b:11059)

[138] Q. Wu, A new exceptional polynomial for the integer transfinite diameter of [0,1], J. Théor. Nombres Bordeaux 15 (2003), 847-861. MR2142240(2006a:11034)

Department of Mathematics, Texas A\&M University, College Station, Texas 77843

E-mail address: terdelyi@math.tamu.edu

Received 14/APR/2008

Originally published in English 